\title{
1 A primer genetic toolkit for exploring mitochondrial biology and disease using
}

\section{2 zebrafish}

3 Ankit Sabharwal ${ }^{1}$, Jarryd M. Campbell ${ }^{1,2}$, Zachary WareJoncas $^{1}$, Mark Wishman ${ }^{1}$, Hirotaka

$4 \quad$ Ata $^{1,2}$, Wiebin Liu ${ }^{1.3}$, Noriko Ichino ${ }^{1}$, Jake D. Bergren ${ }^{1}$, Mark D. Urban ${ }^{1}$, Rhianna Urban ${ }^{1}$, Tanya

5 L. Poshusta ${ }^{1}$, Yonghe Ding ${ }^{1,3}$, Xiaolei Xu ${ }^{1,3}$, Karl J. Clark ${ }^{1,2}$, Stephen C. Ekker ${ }^{1,2^{*}}$

6 1. Department of Biochemistry and Molecular Biology, Mayo Clinic, Rochester, MN 55905,

7 USA

8 2. Center for Clinical and Translational Sciences, Mayo Clinic, Rochester, MN 55905, USA

9 3. Division of Cardiovascular Diseases, Mayo Clinic, Rochester, MN 55905, USA

$10 *$ Corresponding author: Ekker.Stephen@mayo.edu

\section{Keywords:}

12 Mitochondria, Mitochondrial disorders, Zebrafish, gene editing,

13 TALEN, Gene Breaking Transposon 


\section{Abstract}

15 Mitochondria are a dynamic eukaryotic innovation that play diverse roles in biology and disease.

16 The mitochondrial genome is remarkably conserved in all vertebrates, encoding the same 37

17 gene set and overall genomic structure ranging from 16,596 base pairs (bp) in the teleost

18 zebrafish (Danio rerio) to 16,569 bp in humans. Mitochondrial disorders are amongst the most

19 prevalent inherited diseases affecting roughly 1 in every 5000 individuals. Currently, few

20 effective treatments exist for those with mitochondrial ailments, representing a major unmet

21 patient need. Mitochondrial dysfunction is also implicated to be a common component of a wide

22 variety of other human illnesses ranging from neurodegenerative disorders like Huntington's

23 disease and Parkinson's disease to autoimmune illnesses such as multiple sclerosis and

24 rheumatoid arthritis. The electron transport chain (ETC) component of mitochondria is critical

25 for mitochondrial biology and defects can lead to many mitochondrial disease symptoms. Here

26 we present a publicly available collection of genetic mutants created in highly conserved,

27 nuclear-encoded mitochondrial genes in Danio rerio. The zebrafish system represents a

28 potentially powerful new opportunity for the study of mitochondrial biology and disease due to

29 the large number of orthologous genes shared with humans and the many advanced features of this model system from genetics to imaging. This collection includes 22 mutant lines in 18

31 different genes created by locus-specific gene editing to induce frameshift or splice acceptor

32 mutations leading to predicted protein truncation during translation. Also included are 6 lines

33 created by the random insertion of the gene-breaking transposon (GBT) protein trap cassette. All

34 of these targeted mutant alleles truncate conserved domains of genes critical to the proper

35 function of the ETC or genes that have been implicated in human mitochondrial disease. This

36 collection is designed to accelerate the use of zebrafish to study of many different aspects of 
bioRxiv preprint doi: https://doi.org/10.1101/542084; this version posted February 7, 2019. The copyright holder for this preprint (which was not certified by peer review) is the author/funder, who has granted bioRxiv a license to display the preprint in perpetuity. It is made available under aCC-BY-NC-ND 4.0 International license.

37 mitochondrial function with the goal of widening our understanding of their role in biology and

38 human disease. 


\section{Introduction}

40 Mitochondria are semi-autonomous organelles critical for eukaryotic cell function. The

41 mitochondrial endosymbiotic genesis origin hypothesis proposes its evolution from an alpha

42 proteobacterial ancestor, Rickettsia prowazekii [1,2] that were harnessed by a eukaryotic cell as

43 the host billions of years ago [3]. The proteobacterium became a symbiote of the host cell,

44 bringing with it a system for more efficient generation of cellular energy in the form of ATP.

45 During the course of evolution, the genetic material of the mitochondria underwent reductive

46 expansion and was transferred in a retrograde fashion to the nuclear genome. The retrograde

47 movement of genes from the mitochondria to its eukaryotic host paved the way for the

48 mitochondria to specialize as energy production organelles rather than consuming energy

49 repetitively replicating its own multi-copy genome. The mitochondrial-encoded genetic material

50 at present is a vestige of the original proteobacterial genome [4-6] meaning despite having DNA

51 of their own, mitochondria rely heavily on the nuclear genes for most of their functions.

52 Mitochondria have critical functions in metabolism, organ homeostasis, apoptosis and aging.

53 They also play important but still largely mysterious roles in human pathology, as demonstrated

54 by the enormous biological variation and diverse disorders in patients with mitochondrial disease

55 [7-10]. Nearly every organ system can be compromised, but with highly variable and complex

56 physiological and biochemical outcomes. Imaging and basic science of mitochondria showcase

57 how this highly dynamic organelle responds differentially to extrinsic and intrinsic biological

58 signals. However, understanding how mitochondria function in normal biology, and how human

59 mitochondrial DNA variations contribute to health and disease, has been hampered by a lack of

60 effective approaches to manipulate the powerhouse of the cell. 
61 The vertebrate mitochondrial chromosome is circular and includes 37 genes, 13 encoding for

62 protein subunits of the electron transport chain, 22 coding for transfer RNAs, and 2 encoding

63 ribosomal RNAs (Figure 1) [11,12]. The mitochondrial gene order, strand specific nucleotide

64 bias and codon usage is highly conserved [13]. However, mtDNA encoded genes lack introns

65 and utilize a divergent genetic code than their nuclear counterparts $[14,15]$. For instance, AUA

66 codon codes for methionine as per mitochondrial genetic code, whereas the same sequence codes

67 for isoleucine in the nuclear genetic code. Similarly, nuclear stop codon UGA is read as the

68 tryptophan amino acid by the mitochondrial codon cypher.

69 Mitochondria are unique cellular compartments with different DNA and RNA repair and editing

70 rules, hampering attempts at directly manipulating these nucleic acid components. For example,

71 DNA nucleases that introduce double stranded breaks and subsequent repair in nuclear DNA

72 induce the degradation of mtDNA [16-18]. Indeed, none of the canonical DNA repair pathways

73 found in the nucleus have been shown to be active in mitochondria [18-20]. Finally, no system

74 has demonstrated the ability to deliver exogenous DNA or RNA to mitochondria, restricting the

75 tools available for mtDNA editing [21,22]. All of these factors are distinct from the nuclear

76 genome, making mtDNA a far less accessible genome for traditional gene editing methods and

77 reagents.

78 The diverse functions that mitochondria are capable of, including oxidative phosphorylation,

79 would not be possible with the small subset of 13 proteins encoded in mtDNA. Discoveries led

80 by high throughput proteomic approaches have enhanced our knowledge of the mitochondrial

81 proteome [23-27]. There are approximately 1158 nuclear-encoded proteins that localize to the

82 mammalian mitochondria, exerting a dual genetic control via its nuclear counterpart. Nuclear-

83 encoded mitochondrial proteins on the basis of their mitochondrial function can be broadly 
84 classified into different categories including oxidative phosphorylation, energy production,

85 membrane dynamics, genome maintenance, and ion/metabolite homeostasis.

86 Unlike the nuclear genome, where most cells have only two copies, each cell can harbor

87 thousands of copies of mtDNA, depending on environmental needs, making mitochondrial

88 genome engineering a population genetics challenge. When every mtDNA molecule is identical

89 with non-mutagenic variants, the cell is in a genetic state, called homoplasmy, and the

90 mitochondria functions normally. In people with mtDNA-based mitochondrial disease, mtDNA

91 genomes harboring pathogenic mutations typically co-exist with the healthy genomes in a state

92 called heteroplasmy. Homoplasmy may also arise when the cell harbors only mutant mtDNA

93 molecules, resulting in severe clinical manifestations. [28]. The ratio of pathogenic-harboring to

94 nonpathogenic-harboring mtDNA is critical to mitochondrial disease onset. A threshold exists

95 for most mitochondrial disease, at which, the healthy mtDNA genomes can no longer

96 compensate for the pathogenic genomes, resulting in disease onset leading to breakdown of

97 oxidative phosphorylation and other mitochondrial function(s) [29].

98 Mitochondrial disorders are a heterogeneous group of clinical manifestations resulting from

99 either inherited or spontaneous mutations in mtDNA or nDNA leading to altered structure or

100 function of the proteins or RNA that reside in mitochondria $[6,10,30,31]$. The pathophysiology

101 of mitochondrial disorders involves functions of both the nuclear and mitochondrial genomes,

102 conferring additional complexity to the manifestation of the syndromes. The prevalence of 103 mitochondrial disorders is about 1 in 5000 live births; however, the prevalence is often

104 influenced by the presence of founder mutations and consanguinity in populations [31-33].

105 These conditions range in severity dependent on the specific mutation and its prevalence and 
localization within the patient. In addition to diseases specifically progenerated by mitochondrial

107 defects, mitochondrial dysfunction has also been implicated as a cofactor or by-product of many

108 other conditions; including cancer, immune diseases, developmental delays and

109 neurodegenerative condition such as Alzheimer's [34-38].

110 Mitochondrial dysfunction tends to affect primarily high energy systems and can therefore have

111 devastating effects on a wide range of body systems including brain function, liver function,

112 vision, hearing, immune function, and all muscle types [31,39]. The identification and

113 characterization of novel mitochondrial genes in human genetic disorders have also enhanced our

114 knowledge of mitochondrial function [40-44]. However, despite their known prevalence and

115 severity, mitochondrial diseases remain understudied as compared to other genetic conditions,

116 and the options for treatment are limited.

117 The conserved roles of mitochondria have been traditionally uncovered using accessible model

118 systems such as the yeast S.cerevisiae [45-48] and invertebrates including C.elegans [49-52]

119 and D. melanogaster [53-56]. Vertebrate mitochondria, however, are known to have additional

120 innovations and encode unique subunits not present in invertebrate models [5,45,57-67]. Most

121 exploration to date of vertebrate mitochondrial biology has been conducted either in cell culture

122 or in mouse models. However, cells in a dish are missing important environmental cellular and

123 organismal context, whereas mouse mitochondrial experimental work can be hindered by limited

124 population size and invasive imaging techniques.

125 We provide here an initial genetic toolkit for modeling mitochondrial biology and disease in

126 Danio rerio (zebrafish) in an effort to help catalyze the use of this invaluable model organism in

127 mitochondrial research. The zebrafish is a tropical freshwater teleost that has proven to be an

128 invaluable resource for the study of human disease and genetics [68-70]. Principal among the 
many research amenable characteristics of zebrafish are their high genetic orthology to humans, high fecundity, their optical transparency (in the embryonic stage) that lends itself to facile

131 imaging, and the ease of reagent delivery through microinjection into the single celled embryo,

132 which is a full millimeter across before the first mitotic division. Zebrafish also serve as a

133 potentially powerful vertebrate model organism to study human mitochondrial disorders because

134 of conserved mitochondrial genome and mitochondrial genetic machinery. Zebrafish and human 135 mitochondrial chromosomes display $\sim 65 \%$ sequence identity at the nucleotide level, and share 136 the same codon usage, strand specific nucleotide bias and gene order [12] (Figure 1).

137 Over the recent years, zebrafish research has helped shed light on mitochondrial biology and 138 further developed our understanding of the mechanisms of mitochondrial-associated pathology 139 [71-76]. Zebrafish have also been successfully used as a model system to study mitochondrial 140 targeting drugs with implications in development and cardiovascular function $[71,77,78]$. These 141 drugs can simply be dissolved in the water housing the larvae and offer an advantage of large

142 sample size with minimal volumes of drug administered. Drug-screening studies have aided in 143 understanding the pathogenesis of various diseases and helped to identify targets for treatment 144 [77].

145 To help engender an expansion of zebrafish deployment in mitochondrial research, we present a 146 panel or "starter-pack" toolkit of genetic mutants made in nuclear-encoded mitochondrial genes 147 in zebrafish. This mutant panel, which we have named the Marriot Mitochondrial Collection 148 (MMC), consists of 28 zebrafish lines with mutations in 23 different mitochondrial genes. The 149 mutant collection focusses primarily on genes that encode for components of the energy 150 generating electron transport chain with at least one mutant in each complex of the ETC. Other 151 mutants consist of assembly factors, protein chaperones that manage mitochondrial membrane 
152 traffic, and genes related to mitochondrial replication. These mutants were made either by the

153 use of targeted endonuclease [79], or curated from our research group's library of randomly

154 generated insertional mutants [80]. We hope that this collection, in addition to being intrinsically

155 useful, will also help serve as a primer to the modeling of mitochondrial biology and disease in

156 zebrafish.

157 Methods

158 Zebrafish Handling

159 All animal work was conducted under Mayo Clinic's institutional animal welfare approvals

160 (IACUC number: A34513-13-R16).

161 Identification of zebrafish orthologs having putative mitochondrial function

162 A previous study combining discovery and subtractive proteomics with computational,

163 microscopy identified 1098 mouse genes that could encode for proteins residing in mitochondria

164 [24]. They further identified 1013 human orthologs for these genes, providing an initial

165 inventory of the genes coding for proteins resident in mitochondria. Using literature assessment

166 and HUGO database curation approaches, we identified 97 proteins that are involved in the

167 biogenesis and assembly of electron transport chain in mitochondria. Using zebrafish orthologs

168 of human genes from ZFIN (Zebrafish Information Network), 93 zebrafish mitochondrial

169 orthologs were identified. These orthologs were systemically annotated with respect to clinical

170 phenotype by extensive mining from PubMed based published case reports and OMIM database

\section{1 (Supplementary Table 1).}




\section{Mutant Generation}

174 Mutant lines were created by one of two methods, either through the targeted use of

175 Transcription Activator Like Effector Nucleases (TALENs) [79] or by screening Gene Breaking

176 Transposon (GBT) lines for integrations into mitochondrial genes [80]. Reagents were delivered

177 in both methods by the microinjection of either TALEN pairs or GBT transposon and Tol2

178 transposase into single cell zebrafish embryos.

179 TALEN Design/Assembly/Delivery

180 The TALEN mutants in this collection were originally generated as part of a previously 181 published study [79]. In brief, TALEN pairs were designed using the Mojohand software 182 platform [81] (www.talendesign.org) to target highly conserved, and therefore likely functionally 183 important, areas of nuclear-encoded mitochondrial genes. TALEN RVDs were then cloned into 184 pT3Ts-GoldyTALEN (TALEN vector with a T3 transcriptional promoter for in vitro 185 transcription) using the FusX rapid TALEN assembly system [79], which uses the RVD 186 definitions: $\mathrm{HD}=\mathrm{C}, \mathrm{NN}=\mathrm{G}, \mathrm{NI}=\mathrm{A}, \mathrm{NG}=\mathrm{T}$. Following assembly, mRNA was synthesized in vitro 187 using the mMessage Machine T3 kit (Ambion) and extracted by a phenol-chloroform extraction 188 as prescribed in the mMessage Machine manual. The extracted mRNA was then delivered into 189 single cell zebrafish embryos at 100pg doses (50pg per TALEN arm) by microinjection.

\section{TALEN Mutant Screening}

191 Following microinjection, genomic DNA was extracted from F0 larvae three days post 192 fertilization (dpf) by sodium hydroxide extraction. DNA for eight individual larvae was analyzed 193 for NHEJ activity at the TALEN target site by Restriction Fragment Length Polymorphism 194 (RFLP) analysis. Groups with high reported NHEJ activity by RFLP were raised to adulthood 
195 and outcrossed to create an F1 generation. Suspected NHEJ mutants were further verified by

196 Sanger sequencing. F1 larvae demonstrating NHEJ mutations confirmed by both RFLP and

197 Sanger sequencing were raised to adulthood. Fin biopsies were performed on these adults and

198 DNA was extracted by sodium hydroxide extraction.

\section{Gene Breaking Transposon System}

200 The Gene Breaking Transposon System of protein trap system and a complete repository of

201 protocols for the creation and screening of GBT mutant lines has been described [80]. In short,

202 protein trap transposons were delivered in combination with mRNA for Tol2 transposase (25pg

203 each) into single cell zebrafish embryos by microinjection. Embryos were screened for GFP

204 fluorescence at 3-4dpf and classified broadly into three classes [80]. Class three embryos, those

205 with whole body GFP expression, were raised to adulthood and outcrossed to non-transgenic

206 lines to create an F1 generation. mRFP-expressing F1 embryos were sorted by expression

207 pattern, assigned a GBT number, and raised to adulthood. These adult fish were then outcrossed

208 to non-transgenic lines to create an F2 generation upon which all subsequent propagation, testing

209 and imaging was conducted.

210 To determine genes tagged by the protein trap system, rapid amplification of $\underline{\text { cDNA }}$ ends

211 (RACE) was performed as described [82] with minor updates to primer sequences. cDNA was

212 generated using a transposon-specific primer (5R-mRFP-P0) against 250ng of total mRNA in the

213 reverse transcription reaction. PCR was then performed using the following gene-specific

214 primers: 5R-mRFP-P1 and 5R-mRFP-P2. The resulting products were TA cloned for further

215 amplification and then sequence verified for in-frame mRFP fusions by Sanger sequencing. 
216 In some cases, inverse PCR was also conducted to RACE PCR as described [80]. Whole

217 genomic DNA was extracted from individual F2 embryos using a sodium hydroxide extraction

218 and 800ng was digested in a combination reaction using AvrII, NheI, SpeI, and XbaI restriction

219 enzymes. Approximately 200ng of the product of this digestion was self-ligated and used as a

220 template for PCR using the following nested and primary primers. 5' side: 5R-mRFP-P1 and 5R-

221 mRFP-P2 paired with INV-OPT-P1 and INV-OPT-P2, respectively. 3' side: 5R-GFP-P1 and 5R-

222 GFP-P2 with Tol2-ITR(L)-O1 and Tol2-ITR(L)-O3, respectively. The products of the final

223 nested reaction were gel-extracted, cloned, and sequenced. Prospective in frame mRFP fusions

224 were further verified by comparing suspected protein trap allele to GBT expression pattern and

225 by PCR against DNA or cDNA from mRFP carrier siblings versus non-carrier siblings.

226 Protein trap lines as verified by these methods were catalogued for specific genomic fusions

227 using the National Center for Biotechnology Information's (NCBI) Homologene Database.

228 Human orthologs for tagged genes were further identified using blastX searches against the

229 human genome. GBT lines with protein trap fusions to nuclear encoded mitochondrial genes

230 with human orthologues have been included in this collection.

231 In silico analysis to determine protein homology and alteration of DNA sequence by

232 TALENs and GBT in zebrafish mutants

233 Human amino acid sequences were compared to both the zebrafish wild type sequence and each

234 specific zebrafish mutant sequence associated with each allele. Amino acid sequence information

235 for a particular gene was gathered for humans from https://www.uniprot.org/ along with the wild

236 type amino acid sequence for the zebrafish. The first/most common isoform for each entry was

237 used for this analysis. Any added information regarding functional domains or regions was also

238 gathered. A protein-protein BLAST was conducted using https://blast.ncbi.nlm.nih.gov/Blast.cgi 
239 to compare the sequences of the human and wild type zebrafish amino acid sequences. BLAST

240 search settings used included the blastp algorithm. Regions of low and high homology were then

241 mapped out for the sequences.

242 For zebrafish mutant to human comparisons, the cDNA or DNA sequences were gathered via

243 sequencing and put through the translate tool at https://web.expasy.org/translate/. The standard

244 genetic code was used for conversion. Stop codons arising from the frameshifted DNA sequence

245 were found in all mutants. The BLAST analysis conducted on the mutants was taken from the

246 starting methionine to the first encountered stop codon. Regions of low and high homology were

247 then mapped for each sequence.

248 Access to all reported reagents - zebrafish and sequences

249 All listed tools are immediately available through the Mayo Clinic Zebrafish Facility, and all fish

250 lines will be available via ZIRC. Sequences needed for genotyping and related metadata are

251 currently on zfishbook [83].

\section{RESULTS}

\section{Generation of zebrafish mutant collection}

254 We generated zebrafish mutants for a wide selection of nuclear-encoded mitochondrial genes by 255 employing two genetic engineering approaches, gene breaking transposons and TALENs. The 256 collection of zebrafish mutants is referred as the Marriot Mitochondrial Collection (MMC) and 257 comprises of 28 mutants for 23 nuclear-encoded mitochondrial proteins (Figure 2). Out of these,

25822 were created by TALEN indel mutagenesis and 6 were created by gene breaking trap 259 mutagenesis. The mutants include proteins from all known functional pathways involved in 260 mitochondrial homeostasis and ATP generation (Figure 2). 
261 Broadly, the pathways can be classified as subunits of oxidative phosphorylation complexes,

262 chaperones for assembly of oxidative phosphorylation proteins, maintenance proteins for the

263 mitochondrial genome (replication, mtRNA folding and translation), calcium homeostasis and

264 mitochondrial protein import. All of these genes have human orthologs, nearly all with known

265 mutations in which lead to severe clinical manifestations such as Leigh syndrome,

266 cardiomyopathy, progressive external ophthalmoplegia, and oxidative phosphorylation

267 deficiency (Table 1).

268 Table 1: List of nuclear-encoded genes for which zebrafish mutants were generated as part

269 of MMC collection: The current MMC resource list summarizes information on human gene,

270 zebrafish ortholog, mouse ortholog, relevant clinical phenotypes and diseases, protein function,

271 and OMIM ID. Green $=$ Created by TALEN Indel Mutagenesis, Orange $=$ Curated from GBT

272 protein trap lines. Allele designators are highlighted in bold for respective zebrafish paralog.

273 (Chr- Chromosome; OMIM ID: Online Mendelian Inheritance in Man ID; NA-not available).

\begin{tabular}{|c|c|c|c|c|c|c|c|c|c|c|c|}
\hline $\begin{array}{c}\text { Approved } \\
\text { symbol }\end{array}$ & Approved name & $\begin{array}{c}\text { Human } \\
\text { chr }\end{array}$ & $\begin{array}{l}\text { Zebrafish } \\
\text { orthologs }\end{array}$ & $\begin{array}{c}\text { Zebrafish } \\
\text { chr }\end{array}$ & $\begin{array}{c}\text { Allele } \\
\text { designator }\end{array}$ & $\begin{array}{c}\text { Mouse } \\
\text { orthologs }\end{array}$ & $\begin{array}{c}\text { Mouse } \\
\text { chr }\end{array}$ & $\begin{array}{l}\text { Clinical phenotype } \\
\text { observed in humans }\end{array}$ & Disease & $\begin{array}{l}\text { Biological } \\
\text { function }\end{array}$ & $\begin{array}{l}\text { OMIM } \\
\text { ID }\end{array}$ \\
\hline NDUFABI & $\begin{array}{l}\text { NADH:ubiquinone } \\
\text { oxidoreductase } \\
\text { subunit AB1 }\end{array}$ & 16 & $\begin{array}{l}\text { ndufabla } \\
\text { *ndufablb }^{-}\end{array}$ & $\begin{array}{l}1 \\
3\end{array}$ & *mn0135 & Ndufab1b & 7 & -na- & -na- & $\begin{array}{l}\text { Oxidative } \\
\text { phosphorylation } \\
\text { enzymes - } \\
\text { Complex I }\end{array}$ & 603836 \\
\hline NDUFAF6 & $\begin{array}{l}\text { NADH:ubiquinone } \\
\text { oxidoreductase } \\
\text { complex assembly } \\
\text { factor } 6\end{array}$ & 8 & ndufaf6 & 16 & mn0117 & Ndufaf6 & 4 & $\begin{array}{l}\text { Focal right-hand } \\
\text { seizures, ataxia, lactic } \\
\text { acidosis, exercise } \\
\text { intolerance, weakness } \\
\text { and muscle tension }\end{array}$ & $\begin{array}{l}\text { Leigh syndrome, } \\
\text { mitochondrial } \\
\text { complex I } \\
\text { deficiency }\end{array}$ & $\begin{array}{l}\text { Oxidative } \\
\text { phosphorylation } \\
\text { complex } \\
\text { assembly - } \\
\text { Complex I }\end{array}$ & 612392 \\
\hline NDUFS4 & $\begin{array}{l}\text { NADH:ubiquinone } \\
\text { oxidoreductase } \\
\text { subunit S4 }\end{array}$ & 5 & $n d u f s 4$ & 5 & $\mathrm{mn} 0118$ & Ndufs4 & 13 & $\begin{array}{l}\text { Mitochondrial complex } \\
\text { I deficiency }\end{array}$ & $\begin{array}{l}\text { Mitochondrial } \\
\text { complex-I } \\
\text { deficiency }\end{array}$ & $\begin{array}{l}\text { Oxidative } \\
\text { phosphorylation } \\
\text { enzymes - } \\
\text { Complex I }\end{array}$ & 602694 \\
\hline$S D H A$ & $\begin{array}{l}\text { Succinate } \\
\text { dehydrogenase } \\
\text { complex } \\
\text { flavoprotein } \\
\text { subunit A }\end{array}$ & 5 & sdha & 19 & $\mathrm{mn} 0121$ & Sdha & 13 & $\begin{array}{l}\text { Dyspnea, cardiomegaly, } \\
\text { cardiomyopathy, } \\
\text { nystagmus, hypotonia, } \\
\text { gastrointestinal stromal } \\
\text { tumors, } \\
\text { paragangliomas, } \\
\text { pheochromocytoma, } \\
\text { psychomotor regression } \\
\text { and severe } \\
\text { hyperandrogenism }\end{array}$ & $\begin{array}{l}\text { Mitochondrial } \\
\text { complex-II } \\
\text { deficiency; } \\
\text { Cardiomyopathy, } \\
\text { Leigh syndrome; } \\
\text { Paraganglioma }\end{array}$ & $\begin{array}{l}\text { Oxidative } \\
\text { phosphorylation } \\
\text { enzymes - } \\
\text { Complex II }\end{array}$ & 600857 \\
\hline
\end{tabular}


bioRxiv preprint doi: https://doi.org/10.1101/542084; this version posted February 7, 2019. The copyright holder for this preprint (which was not certified by peer review) is the author/funder, who has granted bioRxiv a license to display the preprint in perpetuity. It is made available under aCC-BY-NC-ND 4.0 International license.

\begin{tabular}{|c|c|c|c|c|c|c|c|c|c|c|c|}
\hline $\begin{array}{c}\text { Approved } \\
\text { symbol }\end{array}$ & Approved name & $\begin{array}{c}\text { Human } \\
\text { chr }\end{array}$ & $\begin{array}{l}\text { Zebrafish } \\
\text { orthologs }\end{array}$ & $\begin{array}{c}\text { Zebrafish } \\
\text { chr }\end{array}$ & $\begin{array}{c}\text { Allele } \\
\text { designator }\end{array}$ & $\begin{array}{c}\text { Mouse } \\
\text { orthologs }\end{array}$ & $\begin{array}{c}\text { Mouse } \\
\text { chr }\end{array}$ & $\begin{array}{l}\text { Clinical phenotype } \\
\text { observed in humans }\end{array}$ & Disease & $\begin{array}{c}\text { Biological } \\
\text { function }\end{array}$ & $\begin{array}{c}\text { OMIM } \\
\text { ID }\end{array}$ \\
\hline$B C S 1 L$ & $\begin{array}{l}\text { BCS1 homolog, } \\
\text { ubiquinol- } \\
\text { cytochrome C } \\
\text { reductase complex } \\
\text { chaperone }\end{array}$ & 2 & bcs 11 & 9 & $\mathrm{mn} 0102$ & Bcs11 & 1 & $\begin{array}{l}\text { Growth retardation, } \\
\text { aminoaciduria, } \\
\text { cholestasis, iron } \\
\text { overload, lactic } \\
\text { acidosis, early death, } \\
\text { hypotonia, } \\
\text { encephalopathy, } \\
\text { microcephaly, } \\
\text { psychomotor regression, } \\
\text { hypoglycemia and } \\
\text { hepatic failure }\end{array}$ & $\begin{array}{l}\text { Björnstad } \\
\text { syndrome: } \\
\text { GRACILE } \\
\text { syndrome; Leigh } \\
\text { syndrome; } \\
\text { Mitochondrial } \\
\text { complex III } \\
\text { deficiency, } \\
\text { nuclear type 1 }\end{array}$ & \begin{tabular}{|l|} 
Oxidative \\
phosphorylation \\
complex \\
assembly - \\
Complex III
\end{tabular} & 603647 \\
\hline$U Q C R Q$ & $\begin{array}{l}\text { ubiquinol- } \\
\text { cytochrome c } \\
\text { reductase complex } \\
\text { III subunit VII }\end{array}$ & 5 & uqcrq & 14 & $\begin{array}{l}\mathrm{mn} 0128 \\
\mathrm{mn} 0129\end{array}$ & Uqcrq & 11 & $\begin{array}{l}\text { Severe psychomotor } \\
\text { retardation and } \\
\text { extrapyramidal signs, } \\
\text { dystonia, athetosis, } \\
\text { ataxia, mild axial } \\
\text { hypotonia and marked } \\
\text { global dementia }\end{array}$ & $\begin{array}{l}\text { Mitochondrial } \\
\text { complex III } \\
\text { deficiency }\end{array}$ & $\begin{array}{l}\text { Mitochondrial } \\
\text { complex III: } \\
\text { ubiquinol- } \\
\text { cytochrome c } \\
\text { reductase } \\
\text { complex } \\
\text { subunits }\end{array}$ & 612080 \\
\hline COX10 & $\begin{array}{l}\text { Cytochrome c } \\
\text { oxidase assembly } \\
\text { factor heme A: } \\
\text { farnesyltransferase } \\
\text { COX10 }\end{array}$ & 17 & $\operatorname{cox} 10$ & 12 & $\begin{array}{l}\mathrm{mn} 0107 \\
\mathrm{mn} 0108\end{array}$ & Cox10 & 11 & $\begin{array}{l}\text { Muscle weakness, } \\
\text { hypotonia, ataxia, } \\
\text { ptosis, pyramidal } \\
\text { syndrome, status } \\
\text { epilepticus, lactic } \\
\text { acidosis, hypertrophic } \\
\text { cardiomyopathy, } \\
\text { hypoglycemia and } \\
\text { metabolic acidosis }\end{array}$ & $\begin{array}{l}\text { Leigh syndrome } \\
\text { due to } \\
\text { mitochondrial } \\
\text { COX IV } \\
\text { deficiency; } \\
\text { Mitochondrial } \\
\text { complex IV } \\
\text { deficiency }\end{array}$ & \begin{tabular}{|l|} 
Oxidative \\
phosphorylation \\
complex \\
assembly - \\
Complex IV
\end{tabular} & 602125 \\
\hline COX $4 I 2$ & $\begin{array}{l}\text { Cytochrome C } \\
\text { Oxidase Subunit } \\
4 \mathrm{I} 2\end{array}$ & 20 & $\operatorname{cox} 4 i 2 b$ & 23 & mn0104 & Cox4i2 & 2 & $\begin{array}{l}\text { Exocrine pancreatic } \\
\text { insufficiency, } \\
\text { dyserythropoietic } \\
\text { anemia, and calvarial } \\
\text { hyperostosis }\end{array}$ & $\begin{array}{l}\text { Exocrine } \\
\text { pancreatic } \\
\text { insufficiency, } \\
\text { dyserythropoietic } \\
\text { anemia, and } \\
\text { calvarial } \\
\text { hyperostosis }\end{array}$ & \begin{tabular}{|l|} 
Oxidative \\
phosphorylation \\
complex \\
enzymes - \\
Complex IV
\end{tabular} & 607976 \\
\hline SURF1 & $\begin{array}{l}\text { SURF1, } \\
\text { Cytochrome C } \\
\text { Oxidase Assembly } \\
\text { Factor }\end{array}$ & 9 & surfl & 5 & $\mathrm{mn} 0123$ & Surf1 & 2 & $\begin{array}{l}\text { Childhood onset } \\
\text { neuropathy, lactic } \\
\text { acidosis, mild } \\
\text { intellectual disability } \\
\text { ataxia, facial } \\
\text { dysmorphism, } \\
\text { encephalopathy, } \\
\text { hypotonia, cerebellar } \\
\text { ataxia, deafness, } \\
\text { ophthalmoplegia, } \\
\text { growth retardation and } \\
\text { nystagmus }\end{array}$ & $\begin{array}{l}\text { Charcot-Marie- } \\
\text { Tooth disease, } \\
\text { type 4K; Leigh } \\
\text { syndrome, due to } \\
\text { COX IV } \\
\text { deficiency }\end{array}$ & $\begin{array}{l}\text { Involved in } \\
\text { biogenesis of } \\
\text { cytochrome c } \\
\text { oxidase } \\
\text { complex }\end{array}$ & 185620 \\
\hline ATP5F1E & $\begin{array}{l}\text { ATP synthase F1 } \\
\text { subunit epsilon }\end{array}$ & 20 & atp5fle & 6 & mn0101 & Atp5f1e & 2 & $\begin{array}{l}\text { Neonatal-onset lactic } \\
\text { acidosis, } 3 \text { - } \\
\text { methylglutaconic } \\
\text { aciduria, mild mental } \\
\text { retardation and } \\
\text { peripheral neuropathy }\end{array}$ & $\begin{array}{l}\text { Mitochondrial } \\
\text { complex (ATP } \\
\text { synthase) } \\
\text { deficiency }\end{array}$ & \begin{tabular}{|l|} 
Oxidative \\
phosphorylation \\
complex \\
assembly - ATP \\
synthase
\end{tabular} & 606153 \\
\hline TMEM70 & $\begin{array}{l}\text { Transmembrane } \\
\text { protein } 70\end{array}$ & 8 & tmem70 & 2 & $\begin{array}{l}\mathrm{mn} 0126 \\
\mathrm{mn} 0127\end{array}$ & Tmem70 & 1 & $\begin{array}{l}\text { Lactic acidosis, } \\
\text { encephalopathy, } \\
\text { histiocytoid } \\
\text { cardiomyopathy, } \\
\text { microcephaly, } \\
\text { hypotonia, facial } \\
\text { dysmorphism and 3- } \\
\text { methylglutaconic } \\
\text { aciduria psychomotor } \\
\text { delay and } \\
\text { hyperammonemia }\end{array}$ & $\begin{array}{l}\text { Mitochondrial } \\
\text { complex V (ATP } \\
\text { synthase) } \\
\text { deficiency }\end{array}$ & $\begin{array}{l}\text { Biogenesis of } \\
\text { mitochondrial } \\
\text { ATP synthase }\end{array}$ & 612418 \\
\hline
\end{tabular}


bioRxiv preprint doi: https://doi.org/10.1101/542084; this version posted February 7, 2019. The copyright holder for this preprint (which was not certified by peer review) is the author/funder, who has granted bioRxiv a license to display the preprint in perpetuity. It is made available under aCC-BY-NC-ND 4.0 International license.

\begin{tabular}{|c|c|c|c|c|c|c|c|c|c|c|c|}
\hline $\begin{array}{c}\text { Approved } \\
\text { symbol }\end{array}$ & Approved name & $\begin{array}{c}\text { Human } \\
\text { chr }\end{array}$ & $\begin{array}{l}\text { Zebrafish } \\
\text { orthologs }\end{array}$ & $\begin{array}{c}\text { Zebrafish } \\
\text { chr }\end{array}$ & $\begin{array}{c}\text { Allele } \\
\text { designator }\end{array}$ & $\begin{array}{c}\text { Mouse } \\
\text { orthologs }\end{array}$ & $\begin{array}{c}\text { Mouse } \\
\text { chr }\end{array}$ & $\begin{array}{l}\text { Clinical phenotype } \\
\text { observed in humans }\end{array}$ & Disease & $\begin{array}{l}\text { Biological } \\
\text { function }\end{array}$ & $\begin{array}{c}\text { OMIM } \\
\text { ID }\end{array}$ \\
\hline $\mathrm{COQ} 2$ & $\begin{array}{l}\text { Coenzyme Q2, } \\
\text { Polyprenyltransfe- } \\
\text {-rase }\end{array}$ & 4 & $\operatorname{coq} 2$ & 5 & $\mathrm{mn} 0106$ & Coq2 & 5 & $\begin{array}{l}\text { Multiple system } \\
\text { atrophy, unsteadiness of } \\
\text { gait, nystagmus, gait } \\
\text { ataxia, dysarthria, } \\
\text { speech difficulty, } \\
\text { dysmetria, lactic } \\
\text { acidosis, urinary } \\
\text { dysfunction and } \\
\text { nystagmus }\end{array}$ & $\begin{array}{l}\text { Coenzyme Q10 } \\
\text { deficiency, } \\
\text { primary; Multiple } \\
\text { system atrophy }\end{array}$ & $\begin{array}{l}\text { Biosynthesis of } \\
\text { CoQ, coenzyme } \\
\text { in mitochondrial } \\
\text { respiratory } \\
\text { chain }\end{array}$ & 609825 \\
\hline PDSS2 & $\begin{array}{l}\text { Decaprenyl } \\
\text { diphosphate } \\
\text { synthase subunit } 2\end{array}$ & 6 & $p d s s 2$ & 13 & $\mathrm{mn} 0120$ & Pdss2 & 10 & $\begin{array}{l}\text { Hypotonia, seizures, } \\
\text { cortical blindness, lactic } \\
\text { acidosis, } \\
\text { encephalopathy and } \\
\text { nephrotic syndrome }\end{array}$ & $\begin{array}{l}\text { Coenzyme Q10 } \\
\text { deficiency }\end{array}$ & $\begin{array}{l}\text { Involved in } \\
\text { biosynthesis of } \\
\text { coenzyme Q }\end{array}$ & 610564 \\
\hline MICUI & $\begin{array}{l}\text { Mitochondrial } \\
\text { calcium uptake } 1\end{array}$ & 10 & micul & 13 & $\mathrm{mn} 0132$ & Micu1 & 10 & \begin{tabular}{|l|} 
Proximal muscle \\
weakness and learning \\
disabilities
\end{tabular} & $\begin{array}{l}\text { Myopathy with } \\
\text { extrapyramidal } \\
\text { signs }\end{array}$ & $\begin{array}{l}\text { Regulator of } \\
\text { mitochondrial } \\
\text { calcium uptake }\end{array}$ & 605084 \\
\hline SMDTI & $\begin{array}{l}\text { Single-pass } \\
\text { membrane protein } \\
\text { with aspartate rich } \\
\text { tail }\end{array}$ & 22 & $\begin{array}{l}\text { smdtla } \\
\text { *smdtlb }^{*}\end{array}$ & $\begin{array}{l}3 \\
1\end{array}$ & *mn0122 & Smdt 1 & 15 & -na- & -na- & $\begin{array}{l}\text { Core regulatory } \\
\text { subunit of } \\
\text { calcium channel } \\
\text { in mitochondrial } \\
\text { inner membrane }\end{array}$ & 615588 \\
\hline$M C U$ & $\begin{array}{l}\text { Mitochondrial } \\
\text { calcium uniporter }\end{array}$ & 10 & $m c u$ & 13 & $\mathrm{mn} 0111$ & Mcu & 10 & -na- & -na- & \begin{tabular}{|l} 
Calcium \\
transporter and \\
mediates \\
calcium uptake \\
in mitochondria
\end{tabular} & 614197 \\
\hline TK2 & $\begin{array}{l}\text { Thymidine kinase } \\
2 \text {, mitochondrial }\end{array}$ & 16 & $t k 2$ & 7 & $\mathrm{mn} 0125$ & Tk2 & 8 & $\begin{array}{l}\text { Infantile onset fetal } \\
\text { encephalomyopathy, } \\
\text { failure to thrive, muscle } \\
\text { weakness, mild facial } \\
\text { weakness, myopathy, } \\
\text { axonal neuropathy, } \\
\text { brain atrophy, } \\
\text { hypertrophic } \\
\text { cardiomyopathy, } \\
\text { regression of motor } \\
\text { development, } \\
\text { psychomotor arrest, } \\
\text { dysphonia, dysphagia, } \\
\text { ptosis, impaired gait, } \\
\text { bilateral ptosis, } \\
\text { hypotonia, lactic } \\
\text { acidosis and epilepsy }\end{array}$ & $\begin{array}{l}\text { Progressive } \\
\text { external } \\
\text { ophthalmoplegia; } \\
\text { Mitochondrial } \\
\text { DNA depletion } \\
\text { syndrome 2 } \\
\text { (myopathic type) }\end{array}$ & $\begin{array}{l}\text { Required for } \\
\text { mitochondrial } \\
\text { DNA synthesis }\end{array}$ & 188250 \\
\hline MTFMT & $\begin{array}{l}\text { Mitochondrial } \\
\text { methionyl-tRNA } \\
\text { formyltransferase }\end{array}$ & 15 & $m t f m t$ & 7 & $\begin{array}{l}\mathrm{mn} 0113 \\
\mathrm{mn} 0114\end{array}$ & Mtfmt & 9 & $\begin{array}{l}\text { Psychomotor } \\
\text { developmental delay, } \\
\text { renal dysplasia, mild } \\
\text { facial dysarthria and } \\
\text { ataxia }\end{array}$ & $\begin{array}{l}\text { Combined } \\
\text { oxidative } \\
\text { phosphorylation } \\
\text { deficiency }\end{array}$ & $\begin{array}{l}\text { Catalyzes the } \\
\text { formylation of } \\
\text { methionyl- } \\
\text { tRNA }\end{array}$ & 611766 \\
\hline$M C U$ & $\begin{array}{l}\text { Mitochondrial } \\
\text { calcium uniporter }\end{array}$ & 10 & mси & 13 & $\mathrm{mn} 0599 \mathrm{gt}$ & Mcu & 10 & -na- & -na- & \begin{tabular}{|l} 
Calcium \\
transporter and \\
mediates \\
calcium uptake \\
in mitochondria
\end{tabular} & 614197 \\
\hline LRPPRC & $\begin{array}{l}\text { Leucine rich } \\
\text { pentatricopeptide } \\
\text { repeat containing }\end{array}$ & 2 & lrpprc & 13 & $\mathrm{mn} 0235 \mathrm{gt}$ & Lrpprc & 17 & $\begin{array}{l}\text { Delayed psychomotor } \\
\text { development, mental } \\
\text { retardation, mild } \\
\text { dysmorphic facial } \\
\text { features, hypotonia, } \\
\text { ataxia,development of } \\
\text { lesions in the brainstem } \\
\text { and basal ganglia, } \\
\text { seizures, dysphagia and } \\
\text { hypertrophic } \\
\text { cardiomyopathy }\end{array}$ & $\begin{array}{l}\text { Leigh syndrome, } \\
\text { French-Canadian } \\
\text { type }\end{array}$ & $\begin{array}{l}\text { Involved in } \\
\text { translation of } \\
\text { mitochondrial } \\
\text { encoded cox } \\
\text { subunits and } \\
\text { mediation of } \\
\text { folding of } \\
\text { mitochondrial } \\
\text { transcriptome }\end{array}$ & 607544 \\
\hline
\end{tabular}




\begin{tabular}{|c|c|c|c|c|c|c|c|c|c|c|c|}
\hline $\begin{array}{c}\text { Approved } \\
\text { symbol }\end{array}$ & Approved name & $\underset{\text { chr }}{\text { Human }}$ & $\begin{array}{l}\text { Zebrafish } \\
\text { orthologs }\end{array}$ & $\begin{array}{c}\text { Zebrafish } \\
\text { chr }\end{array}$ & $\begin{array}{c}\text { Allele } \\
\text { designator }\end{array}$ & $\begin{array}{c}\text { Mouse } \\
\text { orthologs }\end{array}$ & $\begin{array}{c}\text { Mouse } \\
\text { chr }\end{array}$ & $\begin{array}{l}\text { Clinical phenotype } \\
\text { observed in humans }\end{array}$ & Disease & $\begin{array}{l}\text { Biological } \\
\text { function }\end{array}$ & $\underset{\text { OMIM }}{\text { ID }}$ \\
\hline MRPSI8B & \begin{tabular}{|l|} 
Mitochondrial \\
ribosomal protein \\
S18B
\end{tabular} & 6 & mrps $18 b$ & 19 & $\mathrm{mn} 0425 \mathrm{gt}$ & Mrps18b & 17 & -na- & -na- & $\begin{array}{l}\text { Part of small } \\
\text { 28S subunit of } \\
\text { mitoribosome }\end{array}$ & 611982 \\
\hline TIMM50 & \begin{tabular}{|l|} 
Translocase of \\
inner mitochondrial \\
membrane 50
\end{tabular} & 19 & timm50 & 15 & mn0906gt & Timm50 & 7 & $\begin{array}{l}\text { Severe intellectual } \\
\text { disability, seizure and 3- } \\
\text { methylglutaconic } \\
\text { aciduria }\end{array}$ & $\mid \begin{array}{l}3- \\
\text { methylglutaconic } \\
\text { aciduria, type IX; } \\
\text { Mitochondrial } \\
\text { complex V } \\
\text { deficiency }\end{array}$ & \begin{tabular}{|l|} 
Subunit of \\
TIM23 inner \\
mitochondrial \\
membrane \\
complex and \\
recognizes \\
mitochondrial \\
targeting signal \\
or pre-sequence
\end{tabular} & 607381 \\
\hline$O G D H$ & \begin{tabular}{|l} 
Oxoglutarate \\
dehydrogenase
\end{tabular} & 7 & $\begin{array}{l}\text { ogdha } \\
\text { *ogdhb }\end{array}$ & $\begin{array}{c}8 \\
10\end{array}$ & *mn0281gt & Ogdh & 11 & Colorectal cancer & $\begin{array}{l}\text { Alpha- } \\
\text { ketoglutarate } \\
\text { dehydrogenase } \\
\text { deficiency }\end{array}$ & \begin{tabular}{|l|} 
Catalyzes the \\
conversion of 2- \\
oxoglutarate to \\
succinyl-CoA \\
and $\mathrm{CO}_{2}$
\end{tabular} & 613022 \\
\hline IDH2 & $\begin{array}{l}\text { Isocitrate } \\
\text { dehydrogenase } \\
(\mathrm{NADP}(+)) 2, \\
\text { mitochondrial }\end{array}$ & 15 & $i d h 2$ & 18 & $\mathrm{mn} 0268 \mathrm{gt}$ & Idh2 & 7 & \begin{tabular}{|l|} 
Acute myeloid leukemia \\
and abnormal \\
production of D-2- \\
hydroxyglutaric acid
\end{tabular} & $\begin{array}{l}\text { D-2- } \\
\text { hydroxyglutaric } \\
\text { aciduria } 2\end{array}$ & $\begin{array}{l}\text { Catalyzes the } \\
\text { conversion of } \\
\text { isocitrate to 2- } \\
\text { oxoglutarate }\end{array}$ & 147650 \\
\hline
\end{tabular}

TALEN- and GBT-mediated targeting of nuclear-encoded mitochondrial genes

276 Amino acid analysis using protein-protein BLAST functions between the wild type human, wild

277 type zebrafish, and mutant zebrafish sequences showed consistent results between the three

278 conditions. The comparison of wild type human and wild type zebrafish sequences showed high

279 areas of homology following the mitochondrial targeting domain in almost every gene, with 80-

$280100 \%$ similarity in catalytic or active domains of the transcripts. Analysis of the mutant zebrafish

281 and human wild type comparison showed a range of difference with predicted frameshift

282 mutations leading to truncation of the protein very early in the transcript (Figure 3A-X). Of the

283 alleles created by TALEN mutagenesis in the MMC collection, all but one (micul) showed a

284 predicted frameshift mutation leading to a truncation event immediately following the NHEJ-

285 mediated insertion or deletion. The GBT mutants showed high levels of homology prior to the

286 transposon integration site followed by a nearly complete loss of normal transcript levels

287 following splicing into the GBT cassette. 


\section{Discussion}

290 In a multicellular organism, each cell is able to carry out its functions due to well-orchestrated

291 cross-talk between the nuclear and mitochondrial genomes. Many mitochondrial functions like

292 energy production, genome maintenance, ion/metabolite homeostasis, membrane dynamics and

293 transport of biomolecules can be attributed to approximately 1158 known nuclear proteins

294 residing in the mitochondria [24,27]. Due to advancements in proteomic technologies in recent

295 years, there has been a surge in documentation of many characterized and uncharacterized

296 mitochondrial proteins. However, correlation between mitochondrial localization of these

297 proteins and their physiological significance in disease progression remains largely unexplored.

298 Out of the 1158 nuclear encoded proteins, only 245 have functional evidence in mitochondrial

299 clinical manifestations [39]. For the remaining 913 proteins, mitochondrial involvement in

300 disease progression has yet to be demonstrated. The obstacles in mitochondrial genetic research,

301 and thus delays in finding effective treatments, are primarily due to the limited tools available to

302 mitochondrial researchers, specifically the small number of available model systems and

303 animals.

304 Most mitochondrial research thus far has made use of model systems that each present their own 305 unique challenge to accurate study of human disease. Yeast, one of the most common laboratory 306 eukaryotes, have been incredibly useful in mitochondrial research, but unlike humans and other 307 animals they lack complex I [45]. Complex 1 deficiency is the most frequent among 308 mitochondrial disorders caused by mutations in 28 out of 48 genes contributing to its assembly 
and biogenesis [84]. Also, complex 1 is responsible for the most ROS generation, which acts as a

310 cue in various signaling pathways [85].

311 Human cell culture has been valuable in exploring mitochondrial disease models, principally

312 through cytoplasmic hybrids that are made by replacing the mitochondria of an immortalized cell

313 line with mutated patient mitochondria [86-89]. However, while these cells are a more faithful

314 representation of human mitochondrial activity than yeast, they have different barriers to

315 accuracy. First, immortalized cells, and cultured cells in general, often have modified metabolic

316 pathways compared to cells in vivo. Second, as is common to all cell culture research, cells in a

317 dish cannot accurately represent the complex interactions and systems biology inherent to a

318 complete organism.

319 In this study, we aim to support the establishment of the zebrafish as a complementary animal

320 model for understanding the role of nuclear-encoded mitochondrial proteins in biology and the

321 pathophysiology of mitochondrial disorders. We propose that zebrafish can be an excellent

322 model organism to study mitochondrial biology primarily because of their conserved genome,

323 codon bias and synteny. Other advantages include their amenability to genetic manipulation and

324 optical clarity, which facilitates direct observation. New gene editing techniques such as

325 TALENs and CRISPRs have aided in the development of humanized disease models in

326 organisms such as zebrafish, mice, and even pigs [90-97].

327 The MMC collection described here encompasses mutations in 23 different nuclear-encoded

328 genes related to mitochondrial function enabling a diverse study of the important roles the

329 mitochondria play in cellular biology. The collection includes mutants in all five complexes of

330 the electron transport chain as well as many other crucial pathways related to protein transport,

331 metabolite synthesis, mtDNA replication and expression, and calcium homeostasis. The first set 
332 of mutants were created using custom gene editing to exons coding for critical functional

333 domains of the protein product. These domains were targeted because they shared high levels of

334 homology to their human ortholog. The second group of mutants consisted of fish that had been

335 injected with a protein trap transposon system that truncates the expected protein products as

336 well as sorting and imaging through fluorescent expression patterns in vivo. Included here, we

337 have curated small group of mutants where the GBT integrated into a mitochondrial gene of 338 interest.

339 In recent years zebrafish have been used to understand the pathophysiology of human 340 mitochondrial disorders such as cardiovascular, multisystemic, neurological, and erythropoiesis,

341 to name a few. Studies for loss of function have been carried out to model left ventricular non-

342 compaction, a genetically heterogeneous cardiomyopathy caused by mutations in nicotinamide

343 nucleotide transhydrogenase $(N N T)$ gene $[98,99]$. Transiently suppressing the levels of $n n t$ gene

344 did mirror the symptoms, such as early ventricular malformation and contractility defects,

345 observed in human patients [99]. Transient suppression of $n d u f b 11$ protein in zebrafish results in

346 cardiac anomalies, confirming the role of NDUFB11 in histiocytoid cardiomyopathy

347 pathogenesis [41].

348 Parkinson disease (PD) is another example where parkin and pink knockdown larvae display 349 mitochondrial fragmentation accompanied by accumulation of oxidative species, mimicking the 350 phenotype observed in PD patients. Loss of dendritic arborization and peripheral neurons were 351 also observed in the knockdown animals [72,100-103]. The zebrafish model of Dravet syndrome 352 shows altered metabolism associated with the pathophysiology [104]. Downregulation of the 353 sodium channel, voltage-gated, type I like, alpha b (scnllab) in zebrafish leads to mitochondrial 354 structural abnormalities accompanied by accumulation of oxidative species [104-106]. 
355 Sideroblastic anemia, caused by mutations in mitoferrin ( $m f r n l)$ gene, has been successfully

356 modelled in zebrafish [107]. The elucidation of its role in zebrafish as iron importer does shed

357 some light on iron related defects in progression of anemia, accompanied by mitochondrial

358 dysfunction. Zebrafish erythrocytes are nucleated and possess organelles such as mitochondria

359 [108] in their cytoplasm, making them an ideal model organism to study ribosomopathies and

360 hematological malignancies [109-114]. Mitochondria in erythrocytes may offer valuable insight

361 into novel biological pathways resident in mitochondria.

362 Transient infantile liver failure and mitochondrial deafness, caused by mutations in tRNA 5-

363 methylaminomethyl-2-thiouridylate methyltransferase (TRMU) gene [115] have been

364 phenocopied in zebrafish [116]. Zebrafish trmu mutants exhibit abnormal mitochondrial

365 morphology, deficient oxidative phosphorylation, defects in hair cells and decreases in the steady

366 state levels of mt-tRNAs, resembling the observations made in patients suffering from this

367 syndrome [116].

368 Taking cues from these studies, many of the genes selected here were prioritized on the basis of 369 the mutations and clinical phenotype reported in patients. Detailed phenotypic analyses will be

370 described in subsequent studies. The zebrafish mutants encode for genes that have been involved

371 in myriad of clinical phenotypes such as encephalopathy, nephrotic syndrome, intellectual

372 disability, psychomotor developmental delay, respiratory chain deficiency, lactic acidosis, iron

373 overload, microcephaly, hypertrophic cardiomyopathy, etc. The superficial investigations of the

374 tissue-specific phenotypes associated with, for example, housekeeping nuclear encoded proteins,

375 have to be refined with organelle level investigations. The repository of mutants encoding for

376 proteins involved in respiratory chain biogenesis and assembly offers the potential to understand

377 the moonlighting role of these proteins. Mutations in these proteins are associated with organ- 
378 specific phenotypes such as neurological, paraganglioma, cardiovascular etc. [41,117]. Extensive

379 energy demand by these tissues is one possible explanation for the progression of these 380 manifestations; however, the role of these proteins in organ-specific cellular niches or

381 homeostasis is also a possibility. These models can help to decipher the role of these proteins in

382 the mitochondrial interactome, when studied in vivo. This underpins the utility of this MMC

383 collection in deciphering the role of mitochondrial proteins in tissue-specific biological 384 pathways.

385 Zebrafish are also amenable to small molecule mediated pharmacological modelling of 386 mitochondrial phenotypes. To test hypoxia as a potential protective therapy in mitochondrial

387 disorders, Von Hippel-Lindau $(v h l)$ null mutant zebrafish, when treated with antimycin mediated 388 mitochondrial insult, exhibited improved survival. In addition, FG-4592 was found to improve 389 survival in response to respiratory chain inhibition, possibly due to increase in hypoxia response 390 [118]. A series of ETC complex specific pharmacological inhibitors such as rotenone (complex 391 I), azide (complex IV), oligomycin (complex V) and chloramphenicol (mitochondrial protein 392 translation) have been used to model respiratory chain dysfunction in zebrafish $[78,119,120]$. 393 Zebrafish larvae display a series of phenotypes, such as developmental arrest, when treated with 394 rotenone. Treatment with azide induced decreased heart rate, loss of motor function, inability to 395 respond to tactical stimulation, neurological damage and mortality. Organ specific manifestations 396 such as neurological and behavioral dysfunction have been reported in 6-7 days post fertilization 397 (dpf) zebrafish larvae upon administration of a titred drug concentration [120]. These 398 observations tilt the scales in favor of zebrafish for modeling mitochondrial clinical 399 manifestations. Gaining insights from these studies, our compendium of mutants provides an 
excellent resource to test a large number of biological and chemical modulators as potential therapies for mitochondrial disorders for which treatment remains elusive.

402 Zebrafish offer many unique advantages for in vivo imaging experiments, as compared to their 403 mammalian counterparts. A classical study corroborating this advantage is in vivo imaging of 404 mitochondrial transport in a single axon, as demonstrated by Yang and his colleagues [121]. 405 Seok and his colleagues generated a transgenic zebrafish line, expressing green fluorescent 406 protein fused to mitochondrial localization sequence from cytochrome c oxidase [71]. 407 Mitochondrial function is often measured by various parameters such as estimation of membrane 408 potential, mitochondrial superoxide species and energy production. Superoxide activity and 409 membrane potential in zebrafish has been measured by employing the use of cell permeable 410 chemical probes such as MitoSox [122] and Dihydrorhodamine 123 (DH123) [73], respectively.

411 Zebrafish transgenic lines expressing genetically encoded calcium and oxidation indicators, have 412 also served as an excellent model to measure calcium homeostasis and oxidative status in vivo in 413 models of mechanosensory hair cell damage and death [123]. Constructs such as Mitotimer allow 414 the measurement of mitochondrial turnover, transport, and changes in the redox history of 415 mitochondria during organogenesis events in zebrafish embryos [124]. This transgenic model 416 enables a sweeping picture of the mitochondrial network, helping in studying cellular processes 417 such as mitophagy and apoptosis.

418 Zebrafish have also provided novel mechanistic insights to interrogate the mitochondrial 419 dynamics, to be called as "in-vivo life cycle of mitochondria" in healthy and diseased conditions 420 [124]. One such example is that of Mitofish [74], that recapitulates mitochondrial network 421 biogenesis, unraveling the role of the organelle in cell-type specific niches of different organ 422 systems. Mitofish is a transgenic zebrafish line that fluorescently labels the mitochondria in the 
423 neurons, enabling non-invasive in vivo observation. Advancement in adaptive optics and lattice

424 light sheet microscopy have empowered researchers to look at vibrant and colorful images of

425 organelles in zebrafish. These technologies have been applied in investigating the organellar

426 dynamics in brain during early development and in the eye of an adult zebrafish [125]. These

427 MMMC mutants provide an excellent platform to study mitochondrial remodeling and

428 homeostasis in the progression of various clinical disorders by mapping the mitochondrial wiring

429 of the cell.

430 These zebrafish mutants have been cryopreserved for the purpose of sharing the lines as a

431 resource with the field for expanding the study of mitochondrial genetics and biology. In

432 addition, our template for the creation of mitochondrial mutants in zebrafish should enable the

433 creation of higher animal models of specific variants of interest. For the purpose of accelerating

434 this research, all lines in this project have had their information deposited with ZIRC, and all of

435 the lines have been made available via zfishbook (www.zfishbook.com) [83].

436 The goal of this study is to disseminate the use of zebrafish as a noblesse oblige in the field of

437 mitochondrial biology and medicine, paving way for the development of novel insight for

438 diagnostic and therapeutic strategies. Ultimately, using this collection of mutants we hope to

439 unravel a small part of the mystery that shrouds one of the most crucial organelles in the

440 cell. We hope that this MMMC mutant collection and the primer we provide for adding to it,

441 will help to usher in a new mitochondrial research using the zebrafish.

\section{Acknowledgements}

443 The authors thank Dr. Eric Schon and Dr. Vamsi Mootha for helping select the gene set targeted

444 in this collection, and the following who contributed to the generation of these new zebrafish 
445 lines including Alexandra Cook, Roberto Lopez Cervera, and the Mayo Clinic Zebrafish Facility

446 staff. Gabriel Martinez Galvez helped with paper illustration design. This work was sponsored

447 by the Mayo Foundation, a gift by the Marriott Foundation and by NIH grants DK84567,

448 GM63904 and HG006431 to SCE.

\section{Author Contributions}

450 The paper was written by AS, MW, ZWJ and SCE. Experiments were executed by JMC, MW,

451 HA, NI, MDU, JDB, RMH, WL, YD and TLP with experimental guidance from XX, KJC and

452 SCE. Data analysis was completed by JMC, NI, JDB, MDU, RMH, MW, WL, YD and AS.

\section{References}

454 1. Andersson SG, Zomorodipour A, Andersson JO, Sicheritz-Ponten T, Alsmark UC, 455 Podowski RM, et al. The genome sequence of Rickettsia prowazekii and the origin of 456 mitochondria. Nature. England; 1998;396: 133-140. doi:10.1038/24094

457 2. Muller M, Martin W. The genome of Rickettsia prowazekii and some thoughts on the $458 \quad$ origin of mitochondria and hydrogenosomes. Bioessays. United States; 1999;21: 377-381. doi:10.1002/(SICI)1521-1878(199905)21:5<377::AID-BIES4>3.0.CO;2-W

460 3. Gray MW, Burger G, Lang BF. Mitochondrial evolution. Science. United States; 1999;283: 1476-1481.

462 4. Thorsness PE, Weber ER. Escape and migration of nucleic acids between chloroplasts, 463 mitochondria, and the nucleus. Int Rev Cytol. United States; 1996;165: 207-234.

464 5. Lynch M, Koskella B, Schaack S. Mutation pressure and the evolution of organelle 465 genomic architecture. Science. United States; 2006;311: 1727-1730. 
doi:10.1126/science.1118884

467 6. Vafai SB, Mootha VK. Mitochondrial disorders as windows into an ancient organelle.

$468 \quad$ Nature. England; 2012;491: 374-383. doi:10.1038/nature11707

469 7. Elson JL, Samuels DC, Turnbull DM, Chinnery PF. Random intracellular drift explains

470 the clonal expansion of mitochondrial DNA mutations with age. Am J Hum Genet. United

$471 \quad$ States; 2001;68: 802-806. doi:10.1086/318801

472 8. DiMauro S, Hirano M. Mitochondrial DNA Deletion Syndromes. In: Adam MP, Ardinger

9. Wallace DC. Mitochondrial DNA mutations in disease and aging. Environ Mol Mutagen. United States; 2010;51: 440-450. doi:10.1002/em.20586

476 10. Elson JL, Smith PM, Greaves LC, Lightowlers RN, Chrzanowska-Lightowlers ZMA, 477 Taylor RW, et al. The presence of highly disruptive $16 \mathrm{~S}$ rRNA mutations in clinical samples indicates a wider role for mutations of the mitochondrial ribosome in human disease. Mitochondrion. Netherlands; 2015;25: 17-27. doi:10.1016/j.mito.2015.08.004

480 11. Andrews RM, Kubacka I, Chinnery PF, Lightowlers RN, Turnbull DM, Howell N. Reanalysis and revision of the Cambridge reference sequence for human mitochondrial

483 12. Broughton RE, Milam JE, Roe BA. The complete sequence of the zebrafish (Danio rerio) mitochondrial genome and evolutionary patterns in vertebrate mitochondrial DNA. Genome Res. United States; 2001;11: 1958-1967. doi:10.1101/gr.156801 
United States; 1992;141: 173-216.

488 14. Osawa S, Jukes TH, Watanabe K, Muto A. Recent evidence for evolution of the genetic $489 \quad$ code. Microbiol Rev. United States; 1992;56: 229-264.

490 15. Richter R, Pajak A, Dennerlein S, Rozanska A, Lightowlers RN, Chrzanowska491 Lightowlers ZMA. Translation termination in human mitochondrial ribosomes. Biochem Soc Trans. England; 2010;38: 1523-1526. doi:10.1042/BST0381523

493 16. Bayona-Bafaluy MP, Blits B, Battersby BJ, Shoubridge EA, Moraes CT. Rapid directional shift of mitochondrial DNA heteroplasmy in animal tissues by a mitochondrially targeted restriction endonuclease. Proc Natl Acad Sci U S A. United States; 2005;102: 14392-

497 17. Srivastava S, Moraes CT. Double-strand breaks of mouse muscle mtDNA promote large deletions similar to multiple mtDNA deletions in humans. Hum Mol Genet. England; 2005;14: 893-902. doi:10.1093/hmg/ddi082

18. Nissanka N, Minczuk M, Moraes CT. Mechanisms of Mitochondrial DNA Deletion Formation. Trends Genet. England; 2019; doi:10.1016/j.tig.2019.01.001

502 19. Alexeyev M, Shokolenko I, Wilson G, LeDoux S. The maintenance of mitochondrial DNA integrity--critical analysis and update. Cold Spring Harb Perspect Biol. United States; 2013;5: a012641. doi:10.1101/cshperspect.a012641 (Mosc). United States; 2018;83: 233-249. doi:10.1134/S0006297918030045

507 21. Patananan AN, Wu T-H, Chiou P-Y, Teitell MA. Modifying the Mitochondrial Genome. 
Cell Metab. United States; 2016;23: 785-796. doi:10.1016/j.cmet.2016.04.004

509

510

511

512

513

514

515

516

517

518

519

520

521

522

523

524

525

526

527

528

22. Gammage PA, Moraes CT, Minczuk M. Mitochondrial Genome Engineering: The Revolution May Not Be CRISPR-Ized. Trends Genet. England; 2018;34: 101-110. doi:10.1016/j.tig.2017.11.001

23. Lopez MF, Kristal BS, Chernokalskaya E, Lazarev A, Shestopalov AI, Bogdanova A, et al. High-throughput profiling of the mitochondrial proteome using affinity fractionation and automation. Electrophoresis. Germany; 2000;21: 3427-3440. doi:10.1002/15222683(20001001)21:16<3427::AID-ELPS3427>3.0.CO;2-L

24. Pagliarini DJ, Calvo SE, Chang B, Sheth SA, Vafai SB, Ong S-E, et al. A mitochondrial protein compendium elucidates complex I disease biology. Cell. United States; 2008;134: 112-123. doi:10.1016/j.cell.2008.06.016

25. Elstner M, Andreoli C, Klopstock T, Meitinger T, Prokisch H. The mitochondrial proteome database: MitoP2. Methods Enzymol. United States; 2009;457: 3-20. doi:10.1016/S0076-6879(09)05001-0

26. Smith AC, Robinson AJ. MitoMiner, an integrated database for the storage and analysis of mitochondrial proteomics data. Mol Cell Proteomics. United States; 2009;8: 1324-1337. doi:10.1074/mcp.M800373-MCP200

27. Calvo SE, Clauser KR, Mootha VK. MitoCarta2.0: an updated inventory of mammalian mitochondrial proteins. Nucleic Acids Res. England; 2016;44: D1251-7. doi:10.1093/nar/gkv1003

28. Taylor RW, Turnbull DM. Mitochondrial DNA mutations in human disease. Nat Rev 
Genet. England; 2005;6: 389-402. doi:10.1038/nrg1606

530

531

532

533

534

535

536

537

538

539

540

541

542

543

29. Rossignol R, Faustin B, Rocher C, Malgat M, Mazat J-P, Letellier T. Mitochondrial threshold effects. Biochem J. England; 2003;370: 751-762. doi:10.1042/BJ20021594

30. Chinnery PF. Mitochondrial Disorders Overview. In: Adam MP, Ardinger HH, Pagon RA, Wallace SE, Bean LJH, Stephens K, et al., editors. Seattle (WA); 1993.

31. Gorman GS, Chinnery PF, DiMauro S, Hirano M, Koga Y, McFarland R, et al. Mitochondrial diseases. Nature reviews. Disease primers. England; 2016. p. 16080. doi:10.1038/nrdp.2016.80

32. Skladal D, Halliday J, Thorburn DR. Minimum birth prevalence of mitochondrial respiratory chain disorders in children. Brain. England; 2003;126: 1905-1912. doi:10.1093/brain/awg170

33. Schaefer AM, McFarland R, Blakely EL, He L, Whittaker RG, Taylor RW, et al. Prevalence of mitochondrial DNA disease in adults. Ann Neurol. United States; 2008;63: 35-39. doi:10.1002/ana.21217

34. Wallace DC, Singh G, Lott MT, Hodge JA, Schurr TG, Lezza AM, et al. Mitochondrial DNA mutation associated with Leber's hereditary optic neuropathy. Science. United States; 1988;242: 1427-1430.

35. Swerdlow RH, Wooten GF. A novel deafness/dystonia peptide gene mutation that causes dystonia in female carriers of Mohr-Tranebjaerg syndrome. Ann Neurol. United States; 2001;50: 537-540.

36. Garone C, Tadesse S, Hirano M. Clinical and genetic spectrum of mitochondrial 
neurogastrointestinal encephalomyopathy. Brain. England; 2011;134: 3326-3332. doi:10.1093/brain/awr245

37. Urrutia PJ, Mena NP, Nunez MT. The interplay between iron accumulation, mitochondrial dysfunction, and inflammation during the execution step of neurodegenerative disorders. Front Pharmacol. Switzerland; 2014;5: 38. doi:10.3389/fphar.2014.00038

38. Singh RK, Saini S, Verma D, Kalaiarasan P, Bamezai RNK. Mitochondrial ND5 mutation mediated elevated ROS regulates apoptotic pathway epigenetically in a P53 dependent manner for generating pro-cancerous phenotypes. Mitochondrion. Netherlands; 2017;35: 35-43. doi:10.1016/j.mito.2017.05.001

39. Craven L, Alston CL, Taylor RW, Turnbull DM. Recent Advances in Mitochondrial

Disease. Annu Rev Genomics Hum Genet. United States; 2017;18: 257-275.

40. Ghezzi D, Saada A, D’Adamo P, Fernandez-Vizarra E, Gasparini P, Tiranti V, et al. FASTKD2 nonsense mutation in an infantile mitochondrial encephalomyopathy associated with cytochrome c oxidase deficiency. Am J Hum Genet. United States;

41. Shehata BM, Cundiff CA, Lee K, Sabharwal A, Lalwani MK, Davis AK, et al. Exome sequencing of patients with histiocytoid cardiomyopathy reveals a de novo NDUFB11 mutation that plays a role in the pathogenesis of histiocytoid cardiomyopathy. Am J Med Genet A. United States; 2015;167A: 2114-2121. doi:10.1002/ajmg.a.37138

570 42. Wesolowska M, Gorman GS, Alston CL, Pajak A, Pyle A, He L, et al. Adult Onset Leigh 571 Syndrome in the Intensive Care Setting: A Novel Presentation of a C12orf65 Related 
Mitochondrial Disease. J Neuromuscul Dis. Netherlands; 2015;2: 409-419. doi:10.3233/JND-150121

574 43. Floyd BJ, Wilkerson EM, Veling MT, Minogue CE, Xia C, Beebe ET, et al. Mitochondrial Protein Interaction Mapping Identifies Regulators of Respiratory Chain Function. Mol Cell. United States; 2016;63: 621-632. doi:10.1016/j.molcel.2016.06.033

577 44. Kohda M, Tokuzawa Y, Kishita Y, Nyuzuki H, Moriyama Y, Mizuno Y, et al. A 578 Comprehensive Genomic Analysis Reveals the Genetic Landscape of Mitochondrial Respiratory Chain Complex Deficiencies. PLoS Genet. United States; 2016;12: e1005679. doi:10.1371/journal.pgen.1005679

45. Boumans H, Grivell LA, Berden JA. The respiratory chain in yeast behaves as a single functional unit. J Biol Chem. United States; 1998;273: 4872-4877.

46. Schwimmer C, Rak M, Lefebvre-Legendre L, Duvezin-Caubet S, Plane G, di Rago J-P. Yeast models of human mitochondrial diseases: from molecular mechanisms to drug screening. Biotechnol J. Germany; 2006;1: 270-281. doi:10.1002/biot.200500053

47. Altmann K, Durr M, Westermann B. Saccharomyces cerevisiae as a model organism to study mitochondrial biology: general considerations and basic procedures. Methods Mol Biol. United States; 2007;372: 81-90. doi:10.1007/978-1-59745-365-3_6

48. Denoth Lippuner A, Julou T, Barral Y. Budding yeast as a model organism to study the effects of age. FEMS Microbiol Rev. England; 2014;38: 300-325. doi:10.1111/15746976.12060 
elegans, a pluricellular model organism to screen new genes involved in mitochondrial genome maintenance. Biochim Biophys Acta. Netherlands; 2010;1802: 765-773. doi:10.1016/j.bbadis.2010.05.007

50. Harrington AJ, Hamamichi S, Caldwell GA, Caldwell KA. C. elegans as a model organism to investigate molecular pathways involved with Parkinson's disease. Dev Dyn. United States; 2010;239: 1282-1295. doi:10.1002/dvdy.22231

51. Moreno-Arriola E, Cardenas-Rodriguez N, Coballase-Urrutia E, Pedraza-Chaverri J, Carmona-Aparicio L, Ortega-Cuellar D. Caenorhabditis elegans: A useful model for studying metabolic disorders in which oxidative stress is a contributing factor. Oxid Med

52. Tissenbaum HA. Using C. elegans for aging research. Invertebr Reprod Dev. England; 2015;59: 59-63. doi:10.1080/07924259.2014.940470

53. Sanchez-Martinez A, Luo N, Clemente P, Adan C, Hernandez-Sierra R, Ochoa P, et al. Modeling human mitochondrial diseases in flies. Biochim Biophys Acta. Netherlands; 2006;1757: 1190-1198. doi:10.1016/j.bbabio.2006.05.008

54. Fernandez-Moreno MA, Farr CL, Kaguni LS, Garesse R. Drosophila melanogaster as a model system to study mitochondrial biology. Methods Mol Biol. United States;

611 55. Guo M. Drosophila as a model to study mitochondrial dysfunction in Parkinson's disease. Cold Spring Harb Perspect Med. United States; 2012;2. doi:10.1101/cshperspect.a009944

613 56. Sen A, Cox RT. Fly Models of Human Diseases: Drosophila as a Model for 
Understanding Human Mitochondrial Mutations and Disease. Curr Top Dev Biol. United States; 2017;121: 1-27. doi:10.1016/bs.ctdb.2016.07.001

57. Lightowlers RN, Rozanska A, Chrzanowska-Lightowlers ZM. Mitochondrial protein synthesis: figuring the fundamentals, complexities and complications, of mammalian mitochondrial translation. FEBS Lett. England; 2014;588: 2496-2503. doi:10.1016/j.febslet.2014.05.054

58. Pett W, Lavrov D V. Cytonuclear Interactions in the Evolution of Animal Mitochondrial tRNA Metabolism. Genome Biol Evol. England; 2015;7: 2089-2101. doi:10.1093/gbe/evv124

623 59. Lavrov D V, Pett W. Animal Mitochondrial DNA as We Do Not Know It: mt-Genome Organization and Evolution in Nonbilaterian Lineages. Genome Biol Evol. England;

60. Brown WM, George MJ, Wilson AC. Rapid evolution of animal mitochondrial DNA. Proc Natl Acad Sci U S A. United States; 1979;76: 1967-1971.

628 61. Moyes CD, Suarez RK, Hochachka PW, Ballantyne JS. A comparison of fuel preferences of mitochondria from vertebrates and invertebrates. Can J Zool. NRC Research Press;

62. Grossman LI, Wildman DE, Schmidt TR, Goodman M. Accelerated evolution of the electron transport chain in anthropoid primates. Trends Genet. England; 2004;20: 578585. doi:10.1016/j.tig.2004.09.002 1990;68: 1337-1349. doi:10.1139/z90-201

63. Gershoni M, Fuchs A, Shani N, Fridman Y, Corral-Debrinski M, Aharoni A, et al. 
Coevolution predicts direct interactions between mtDNA-encoded and nDNA-encoded subunits of oxidative phosphorylation complex i. J Mol Biol. England; 2010;404: 158171. doi:10.1016/j.jmb.2010.09.029

64. Haen KM, Pett W, Lavrov D V. Parallel loss of nuclear-encoded mitochondrial aminoacyl-tRNA synthetases and mtDNA-encoded tRNAs in Cnidaria. Mol Biol Evol. United States; 2010;27: 2216-2219. doi:10.1093/molbev/msq112

65. Rea SL, Graham BH, Nakamaru-Ogiso E, Kar A, Falk MJ. Bacteria, yeast, worms, and flies: exploiting simple model organisms to investigate human mitochondrial diseases. Dev Disabil Res Rev. United States; 2010;16: 200-218. doi:10.1002/ddrr.114

66. Kuzmenko A V, Levitskii SA, Vinogradova EN, Atkinson GC, Hauryliuk V, Zenkin N, et al. Protein biosynthesis in mitochondria. Biochemistry (Mosc). United States; 2013;78: 855-866. doi:10.1134/S0006297913080014

67. Zhao J, Lendahl U, Nister M. Regulation of mitochondrial dynamics: convergences and divergences between yeast and vertebrates. Cell Mol Life Sci. Switzerland; 2013;70: 951-976. doi:10.1007/s00018-012-1066-6

68. Lieschke GJ, Currie PD. Animal models of human disease: zebrafish swim into view. Nat Rev Genet. England; 2007;8: 353-367. doi:10.1038/nrg2091

69. Lin C-Y, Chiang C-Y, Tsai H-J. Zebrafish and Medaka: new model organisms for modern biomedical research. J Biomed Sci. England; 2016;23: 19. doi:10.1186/s12929-016-02365

70. Blanco-Sanchez B, Clement A, Phillips JB, Westerfield M. Zebrafish models of human 
eye and inner ear diseases. Methods Cell Biol. United States; 2017;138: 415-467. doi:10.1016/bs.mcb.2016.10.006

71. Kim MJ, Kang KH, Kim C-H, Choi S-Y. Real-time imaging of mitochondria in transgenic zebrafish expressing mitochondrially targeted GFP. Biotechniques. England; 2008;45: 331-334. doi:10.2144/000112909 deficiency and dopaminergic neuronal cell loss in parkin-deficient zebrafish (Danio rerio). Brain. England; 2009;132: 1613-1623. doi:10.1093/brain/awp108

73. Song Y, Selak MA, Watson CT, Coutts C, Scherer PC, Panzer JA, et al. Mechanisms underlying metabolic and neural defects in zebrafish and human multiple acyl-CoA dehydrogenase deficiency (MADD). PLoS One. United States; 2009;4: e8329. doi:10.1371/journal.pone.0008329 of disease-related mitochondrial dynamics in a vertebrate model system. J Neurosci. United States; 2012;32: 16203-16212. doi:10.1523/JNEUROSCI.1327-12.2012

671 75. Steele SL, Prykhozhij S V, Berman JN. Zebrafish as a model system for mitochondrial 672 biology and diseases. Transl Res. United States; 2014;163: 79-98. doi:10.1016/j.trs1.2013.08.008

674 76. Byrnes J, Ganetzky R, Lightfoot R, Tzeng M, Nakamaru-Ogiso E, Seiler C, et al. 675 Pharmacologic modeling of primary mitochondrial respiratory chain dysfunction in zebrafish. Neurochem Int. England; 2017; doi:10.1016/j.neuint.2017.07.008 
677 77. Pinho BR, Santos MM, Fonseca-Silva A, Valentao P, Andrade PB, Oliveira JMA. How

678

679

680

681

682

683

684

685

686

687

688

689

690

691

692

693

694

695

696

697

698

mitochondrial dysfunction affects zebrafish development and cardiovascular function: an in vivo model for testing mitochondria-targeted drugs. $\mathrm{Br} \mathrm{J}$ Pharmacol. England; 2013;169: 1072-1090. doi:10.1111/bph.12186

78. Polyak E, Ostrovsky J, Peng M, Dingley SD, Tsukikawa M, Kwon YJ, et al. Nacetylcysteine and vitamin E rescue animal longevity and cellular oxidative stress in preclinical models of mitochondrial complex I disease. Mol Genet Metab. United States; 2018;123: 449-462. doi:10.1016/j.ymgme.2018.02.013

79. Ma AC, McNulty MS, Poshusta TL, Campbell JM, Martinez-Galvez G, Argue DP, et al. FusX: A Rapid One-Step Transcription Activator-Like Effector Assembly System for Genome Science. Hum Gene Ther. United States; 2016;27: 451-463. doi:10.1089/hum.2015.172

80. Clark KJ, Balciunas D, Pogoda H-M, Ding Y, Westcot SE, Bedell VM, et al. In vivo protein trapping produces a functional expression codex of the vertebrate proteome. Nat Methods. United States; 2011;8: 506-515. doi:10.1038/nmeth.1606

81. Neff KL, Argue DP, Ma AC, Lee HB, Clark KJ, Ekker SC. Mojo Hand, a TALEN design tool for genome editing applications. BMC Bioinformatics. England; 2013;14: 1. doi:10.1186/1471-2105-14-1

82. Clark KJ, Geurts AM, Bell JB, Hackett PB. Transposon vectors for gene-trap insertional mutagenesis in vertebrates. Genesis. United States; 2004;39: 225-233. doi:10.1002/gene.20049

83. Clark KJ, Argue DP, Petzold AM, Ekker SC. zfishbook: connecting you to a world of 
zebrafish revertible mutants. Nucleic Acids Res. England; 2012;40: D907-11.

700 doi:10.1093/nar/gkr957

701

84. Sharma LK, Lu J, Bai Y. Mitochondrial respiratory complex I: structure, function and implication in human diseases. Curr Med Chem. United Arab Emirates; 2009;16: 12661277.

704

85. Liu Y, Fiskum G, Schubert D. Generation of reactive oxygen species by the mitochondrial electron transport chain. J Neurochem. England; 2002;80: 780-787.

86. King MP, Attardi G. Human cells lacking mtDNA: repopulation with exogenous mitochondria by complementation. Science. United States; 1989;246: 500-503.

87. Koga Y, Davidson M, Schon EA, King MP. Analysis of cybrids harboring MELAS 709 mutations in the mitochondrial tRNA(Leu(UUR)) gene. Muscle Nerve Suppl. United

711 88. Vithayathil SA, Ma Y, Kaipparettu BA. Transmitochondrial cybrids: tools for functional States; 1995;3: S119-23.

714 89. Wilkins HM, Carl SM, Swerdlow RH. Cytoplasmic hybrid (cybrid) cell lines as a practical 715 model for mitochondriopathies. Redox Biol. Netherlands; 2014;2: 619-631. doi:10.1016/j.redox.2014.03.006

717 90. Bedell VM, Wang Y, Campbell JM, Poshusta TL, Starker CG, Krug RG 2nd, et al. In vivo genome editing using a high-efficiency TALEN system. Nature. England; 2012;491: 114118. doi:10.1038/nature11537 
720 91. Hwang WY, Fu Y, Reyon D, Maeder ML, Tsai SQ, Sander JD, et al. Efficient genome 721 editing in zebrafish using a CRISPR-Cas system. Nat Biotechnol. United States; 2013;31:

722 227-229. doi:10.1038/nbt.2501

723

724

725

726

727

728

729

730

731

732

733

734

735

736

737

738

739

740

92. Jiang W, Zhou H, Bi H, Fromm M, Yang B, Weeks DP. Demonstration of CRISPR/Cas9/sgRNA-mediated targeted gene modification in Arabidopsis, tobacco, sorghum and rice. Nucleic Acids Res. England; 2013;41: e188. doi:10.1093/nar/gkt780

93. Fujihara Y, Ikawa M. CRISPR/Cas9-based genome editing in mice by single plasmid injection. Methods Enzymol. United States; 2014;546: 319-336. doi:10.1016/B978-0-12801185-0.00015-5

94. Hsu PD, Lander ES, Zhang F. Development and applications of CRISPR-Cas9 for genome engineering. Cell. United States; 2014;157: 1262-1278. doi:10.1016/j.cell.2014.05.010

95. Zhang F, Wen Y, Guo X. CRISPR/Cas9 for genome editing: progress, implications and challenges. Hum Mol Genet. England; 2014;23: R40-6. doi:10.1093/hmg/ddu125

96. Hashimoto M, Bacman SR, Peralta S, Falk MJ, Chomyn A, Chan DC, et al. MitoTALEN: A General Approach to Reduce Mutant mtDNA Loads and Restore Oxidative Phosphorylation Function in Mitochondrial Diseases. Mol Ther. United States; 2015;23: 1592-1599. doi:10.1038/mt.2015.126

97. Niu D, Wei H-J, Lin L, George H, Wang T, Lee I-H, et al. Inactivation of porcine endogenous retrovirus in pigs using CRISPR-Cas9. Science. United States; 2017;357: 1303-1307. doi:10.1126/science.aan4187 
741 98. Meimaridou E, Kowalczyk J, Guasti L, Hughes CR, Wagner F, Frommolt P, et al. Mutations in NNT encoding nicotinamide nucleotide transhydrogenase cause familial glucocorticoid deficiency. Nat Genet. United States; 2012;44: 740-742. doi:10.1038/ng.2299

745

746

747

99. Bainbridge MN, Davis EE, Choi W-Y, Dickson A, Martinez HR, Wang M, et al. Loss of Function Mutations in NNT Are Associated With Left Ventricular Noncompaction. Circ

Cardiovasc $\quad$ Genet. $\quad$ United $\quad$ States; 2015;8: 544-552.

100. Anichtchik O, Diekmann H, Fleming A, Roach A, Goldsmith P, Rubinsztein DC. Loss of PINK1 function affects development and results in neurodegeneration in zebrafish. $\mathrm{J}$ Neurosci. United States; 2008;28: 8199-8207. doi:10.1523/JNEUROSCI.0979-08.2008

101. Fett ME, Pilsl A, Paquet D, van Bebber F, Haass C, Tatzelt J, et al. Parkin is protective against proteotoxic stress in a transgenic zebrafish model. PLoS One. United States; 2010;5: e11783. doi:10.1371/journal.pone.0011783

102. Sallinen V, Kolehmainen J, Priyadarshini M, Toleikyte G, Chen Y-C, Panula P. Dopaminergic cell damage and vulnerability to MPTP in Pink1 knockdown zebrafish. Neurobiol Dis. United States; 2010;40: 93-101. doi:10.1016/j.nbd.2010.06.001

103. Xi Y, Ryan J, Noble S, Yu M, Yilbas AE, Ekker M. Impaired dopaminergic neuron development and locomotor function in zebrafish with loss of pink1 function. Eur $\mathbf{J}$ Neurosci. France; 2010;31: 623-633. doi:10.1111/j.1460-9568.2010.07091.x

104. Kumar MG, Rowley S, Fulton R, Dinday MT, Baraban SC, Patel M. Altered Glycolysis and Mitochondrial Respiration in a Zebrafish Model of Dravet Syndrome. eNeuro. United 
States; 2016;3. doi:10.1523/ENEURO.0008-16.2016

764 105. Baraban SC, Dinday MT, Hortopan GA. Drug screening in Scn1a zebrafish mutant 2013;4: 2410. doi:10.1038/ncomms3410

106. Grone BP, Qu T, Baraban SC. Behavioral Comorbidities and Drug Treatments in a Zebrafish scn1lab Model of Dravet Syndrome. eNeuro. United States; 2017;4. doi:10.1523/ENEURO.0066-17.2017

770

771

772

773

774

775

776

777

778

779

780

781

782

107. Guernsey DL, Jiang H, Campagna DR, Evans SC, Ferguson M, Kellogg MD, et al. Mutations in mitochondrial carrier family gene SLC25A38 cause nonsyndromic autosomal recessive congenital sideroblastic anemia. Nat Genet. United States; 2009;41: 651-653. doi:10.1038/ng.359

108. Bronnimann D, Annese T, Gorr TA, Djonov V. Splitting of circulating red blood cells as an in vivo mechanism of erythrocyte maturation in developing zebrafish, chick and mouse embryos. J Exp Biol. England; 2018;221. doi:10.1242/jeb.184564

109. Berman J, Hsu K, Look AT. Zebrafish as a model organism for blood diseases. Br $\mathbf{J}$ Haematol. England; 2003;123: 568-576.

110. Danilova N, Sakamoto KM, Lin S. Ribosomal protein S19 deficiency in zebrafish leads to developmental abnormalities and defective erythropoiesis through activation of p53 protein family. Blood. United States; 2008;112: 5228-5237. doi:10.1182/blood-2008-01132290

783 111. Butler MG, Gore A V, Weinstein BM. Zebrafish as a model for hemorrhagic stroke. 
Methods Cell Biol. United States; 2011;105: 137-161. doi:10.1016/B978-0-12-381320-

785 6.00006-0

112. Kulkeaw K, Sugiyama D. Zebrafish erythropoiesis and the utility of fish as models of anemia. Stem Cell Res Ther. England; 2012;3: 55. doi:10.1186/scrt146

113. Kretz CA, Weyand AC, Shavit JA. Modeling Disorders of Blood Coagulation in the Zebrafish. Curr Pathobiol Rep. United States; 2015;3: 155-161. doi:10.1007/s40139-0150081-3

114. Avagyan S, Zon LI. Fish to Learn: Insights into Blood Development and Blood Disorders from Zebrafish Hematopoiesis. Hum Gene Ther. United States; 2016;27: 287-294. doi:10.1089/hum.2016.024

115. Prezant TR, Agapian J V, Bohlman MC, Bu X, Oztas S, Qiu WQ, et al. Mitochondrial ribosomal RNA mutation associated with both antibiotic-induced and non-syndromic deafness. Nat Genet. United States; 1993;4: 289-294. doi:10.1038/ng0793-289

116. Zhang Q, Zhang L, Chen D, He X, Yao S, Zhang Z, et al. Deletion of Mtu1 (Trmu) in zebrafish revealed the essential role of tRNA modification in mitochondrial biogenesis and hearing function. Nucleic Acids Res. England; 2018;46: 10930-10945. doi:10.1093/nar/gky758

117. DiMauro S, Schon EA. Mitochondrial respiratory-chain diseases. N Engl J Med. United States; 2003;348: 2656-2668. doi:10.1056/NEJMra022567 therapy for mitochondrial disease. Science. United States; 2016;352: 54-61. 
doi:10.1126/science.aad9642

806

807

808

809

810

811

812

813

814

815

816

817

818

819

820

821

822

823

824

825

119. Bretaud S, Lee S, Guo S. Sensitivity of zebrafish to environmental toxins implicated in Parkinson's disease. Neurotoxicol Teratol. United States; 2004;26: 857-864. doi:10.1016/j.ntt.2004.06.014

120. Byrnes J, Ganetzky R, Lightfoot R, Tzeng M, Nakamaru-Ogiso E, Seiler C, et al. Pharmacologic modeling of primary mitochondrial respiratory chain dysfunction in zebrafish. Neurochem Int. England; 2018;117: 23-34. doi:10.1016/j.neuint.2017.07.008

121. Xu Y, Chen M, Hu B, Huang R, Hu B. In vivo Imaging of Mitochondrial Transport in Single-Axon Regeneration of Zebrafish Mauthner Cells. Front Cell Neurosci. Switzerland; 2017;11: 4. doi:10.3389/fncel.2017.00004

122. Dowling JJ, Arbogast S, Hur J, Nelson DD, McEvoy A, Waugh T, et al. Oxidative stress and successful antioxidant treatment in models of RYR1-related myopathy. Brain. England; 2012;135: 1115-1127. doi:10.1093/brain/aws036

123. Pickett SB, Thomas ED, Sebe JY, Linbo T, Esterberg R, Hailey DW, et al. Cumulative mitochondrial activity correlates with ototoxin susceptibility in zebrafish mechanosensory hair cells. Elife. England; 2018;7. doi:10.7554/eLife.38062

124. Mandal A, Pinter K, Drerup CM. Analyzing Neuronal Mitochondria in vivo Using Fluorescent Reporters in Zebrafish. Front cell Dev Biol. Switzerland; 2018;6: 144. doi:10.3389/fcell.2018.00144

125. Liu T-L, Upadhyayula S, Milkie DE, Singh V, Wang K, Swinburne IA, et al. Observing the cell in its native state: Imaging subcellular dynamics in multicellular organisms. 
bioRxiv preprint doi: httos://doi org/101101/542084: this version posted Februarv 72019 . The copvriaht holder for this preprint (which was not certified by peer review) is the author/funder, who has granted bioRxiv a license to display the preprint in perpetuity. It is made available under aCC-BY-NC-ND 4.0 International license.

Science. United States; 2018;360. doi:10.1126/science.aaq1392

827 
Figures Legends:

829

830

831

832

833

834

835

836

837

838

839

840

841

842

843

Figure 1: Circular representation of the zebrafish (NC_002333.2) and human mitochondrial genomes (NC_012920.1): Both genomes share the same synteny, number of genes and are nearly identical in size.

Figure 2: Zebrafish Marriot mitochondrial mutant collection: Schematic representation of various mitochondrial resident pathways for which zebrafish mutants were generated. The nuclear-encoded mitochondrial proteins have been illustrated according to the function they are involved in mitochondrial maintenance and homeostasis. Mutants generated by gene editing are depicted as grey, whereas those generated by the GBT system are depicted in pink.

Figure 3: A depiction of homology of the mutants created for proteins involved in different mitochondrial resident pathways: A-C: Complex 1 of mitochondrial respiratory chain; A: NADH: ubiquinone oxidoreductase subunit AB1 b (Ndufab1b); B: NADH dehydrogenase association factor 6 (Ndufaf6); C: NADH dehydrogenase iron sulfur protein 4 (Ndufs4); D: Complex 2 of mitochondrial respiratory chain, Succinate dehydrogenase subunit A (Sdha), subunit; E-F: Complex 3 of mitochondrial respiratory chain; E: Mitochondrial chaperone protein BCS1 (Bcs11); F: Ubiquinol-cytochrome c reductase subunit VIII (Uqcrq); G-I: Complex 4 of mitochondrial respiratory chain; G: Protoheme IX farnesyltransferase (Cox10); H: Cytochrome c oxidase IV subunit 1 (Cox4i2b); I: Surfeit locus protein 1 (Surf1); J-K: Complex 5 of mitochondrial respiratory chain; J: ATP synthase subunit epsilon (Atp5e); K: Transmembrane protein 70 (Tmem70); L-M: Biosynthesis of coenzyme Q; L: 4hydroxybenzoate polyprenyltransferase (Coq2) M: Decaprenyl-disphosphate synthase subunit 2 (Pdss2); N-Q: Mitochondrial calcium homeostasis; N: Mitochondrial calcium uptake protein 1 
850 (Micu1); O: Calcium uniporter protein (Mcu) generated by TALEN and P; GBT; Q: Essential

851 MCU regulator a (Smdt1a); R-U: Mitochondrial genome maintenance; R: Thymidine kinase 2

852 (Tk2); S: Leucine-rich pentatricopeptide repeat-containing (Lrpprc); T: Mitochondrial

853 methionyl-tRNA formyltransferase (Mtfmt); U: 28s Ribosomal protein s18b, mitochondrial

854 (Mrps18b); V: Mitochondrial protein import; Mitochondrial import inner membrane

855 translocase subunit TIM50 (Timm50); W-X: Mitochondrial metabolite synthesis; W: 2-

856 Oxoglutarate dehydrogenase b (Ogdhb); X: Isocitrate dehydrogenase (Idh2). 
bioRxiv preprint doi: https://doi.org/10.1101/542084; this version posted February 7,2019 . The copyright holder for this preprint (which was not certified by peer review) is the author/funder, who has granted bioRxiv a license to display the preprint in perpetuity. It is made available under aCC-BY-NC-ND 4.0 International license.

\section{Figures:}

\section{Figure 1:}
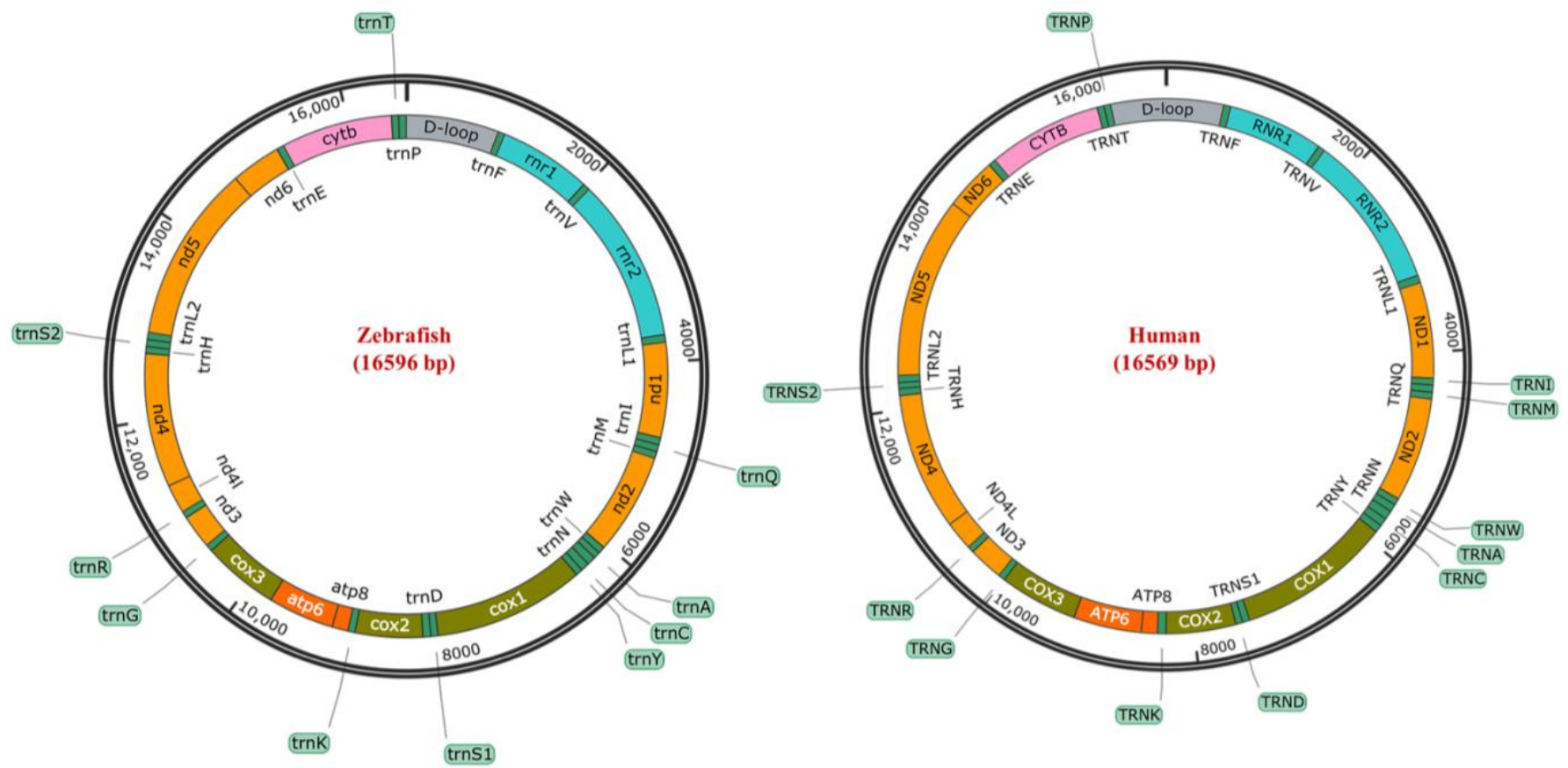

860 
bioRxiv preprint doi: https://doi.org/10.1101/542084; this version posted February 7, 2019. The copyright holder for this preprint (which was not certified by peer review) is the author/funder, who has granted bioRxiv a license to display the preprint in perpetuity. It is made available under aCC-BY-NC-ND 4.0 International license.

\section{Figure 2:}

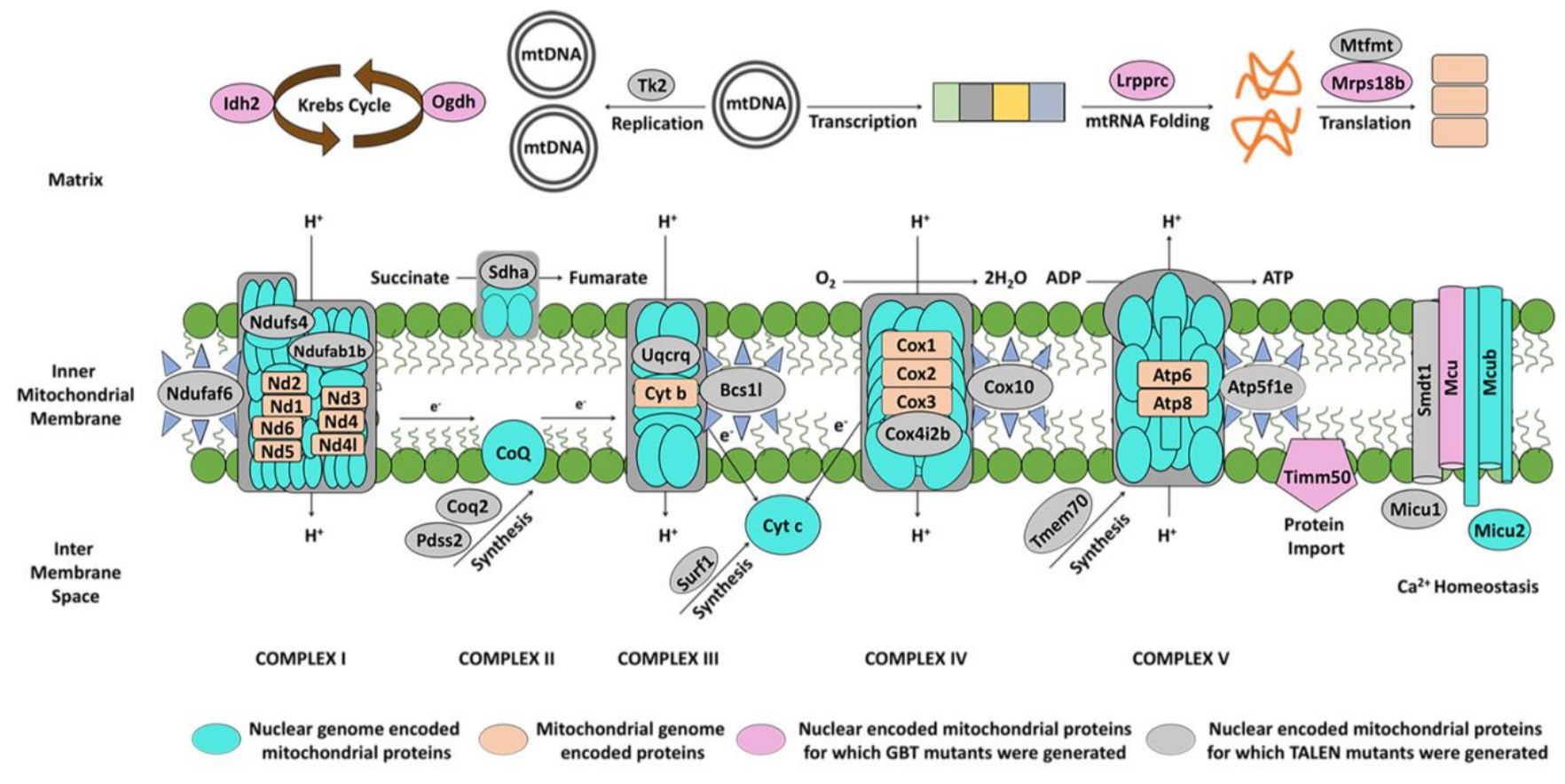


bioRxiv preprint doi: https://doi.org/101101/542084; this version posted February 7,2019 . The copyright holder for this preprint (which was not certified by peer review) is the author/funder, who has granted bioRxiv a license to display the preprint in perpetuity. It is made available under aCC-BY-NC-ND 4.0 International license.

\section{Figure 3:}

Amino Acid Homology $\quad 0-40 \% \square 40-60 \%$

A

NADH: ubiquinone oxidoreductase subunit AB1 (Ndufab1b) Human:

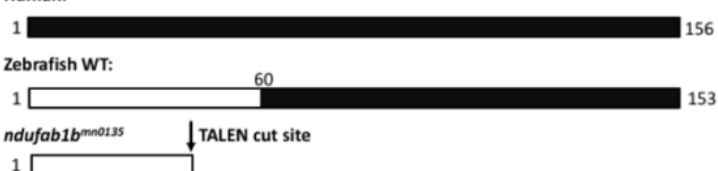

C

NADH dehydrogenase iron sulfur protein 4 (Ndufs4)

Human: $\quad$ Functional domain

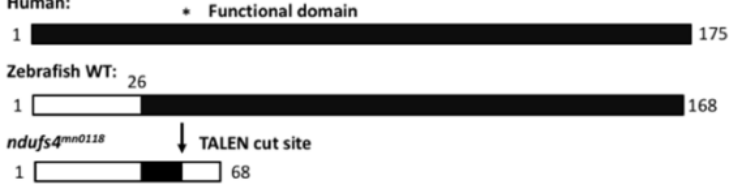

$\mathbf{E}$

Mitochondrial chaperone protein BCS1 (Bcs1I)

Human:

DNA binding domain

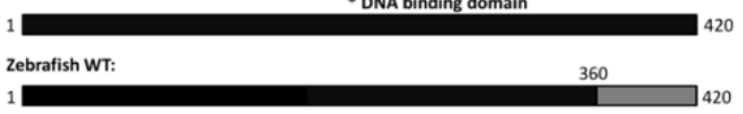

bcs1/mno102 $\downarrow$ TALEN cut site

$1 \square 202$

G

Protoheme IX farnesyltransferase (Cox10)

Human:

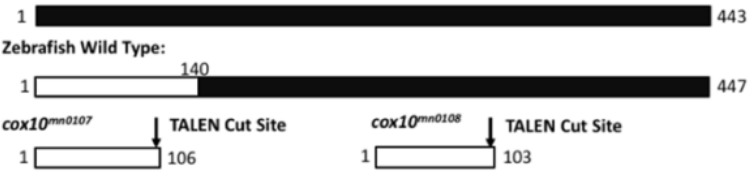

I

Surfeit locus protein 1 (Surf1)

Human:

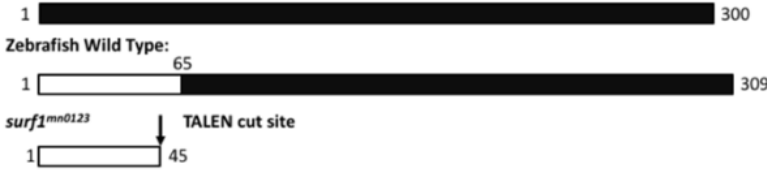

$\mathbf{K}$

Transmembrane protein 70 (Tmem70)

Human:

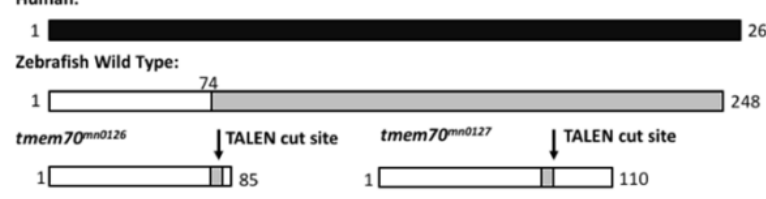

$60-80 \% \square 80-100 \%$

B

NADH dehydrogenase, association factor 6 (Ndufaf6)

Human: * Functional domain

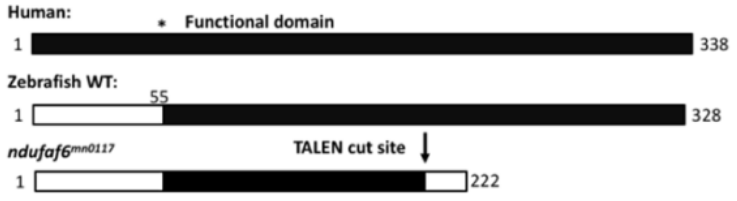

D

Succinate dehydrogenase subunit A (Sdha)

Human WT: * Catalytic site

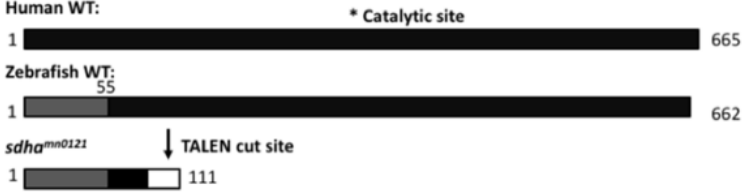

$\mathbf{F}$

Ubiquinol-cytochrome c reductase, subunit VIII (Uqcrq) Human:

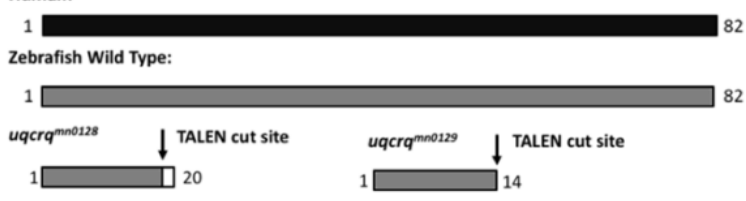

H Cytochrome c oxidase IV subunit 1 (Cox4i2b)

Human:

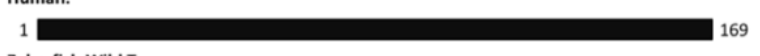

Zebrafish Wild Type:

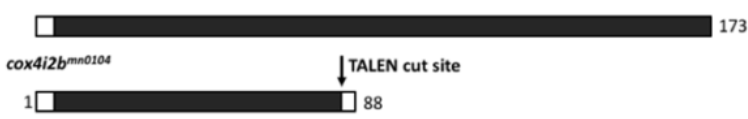

ATP synthase subunit epsilon (Atp5e)

Human:

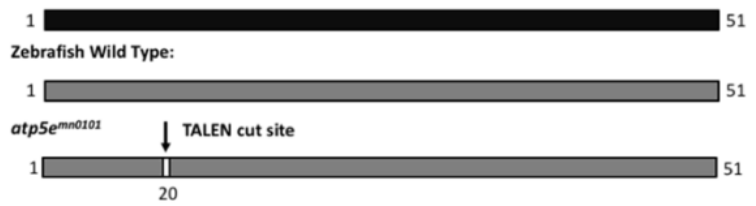

4-hydroxybenzoate polyprenyltransferase (Coq2)

Human:

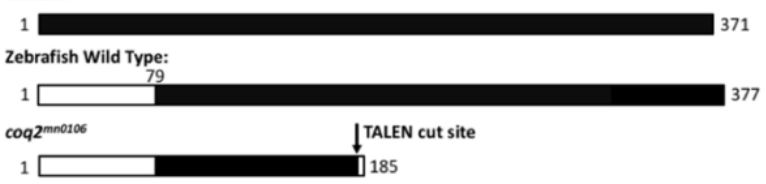


bioRxiv preprint doi: https://doi.org/101101/542084; this version posted February 7,2019 . The copyright holder for this preprint (which was not certified by peer review) is the author/funder, who has granted bioRxiv a license to display the preprint in perpetuity. It is made available under aCC-BY-NC-ND 4.0 International license.

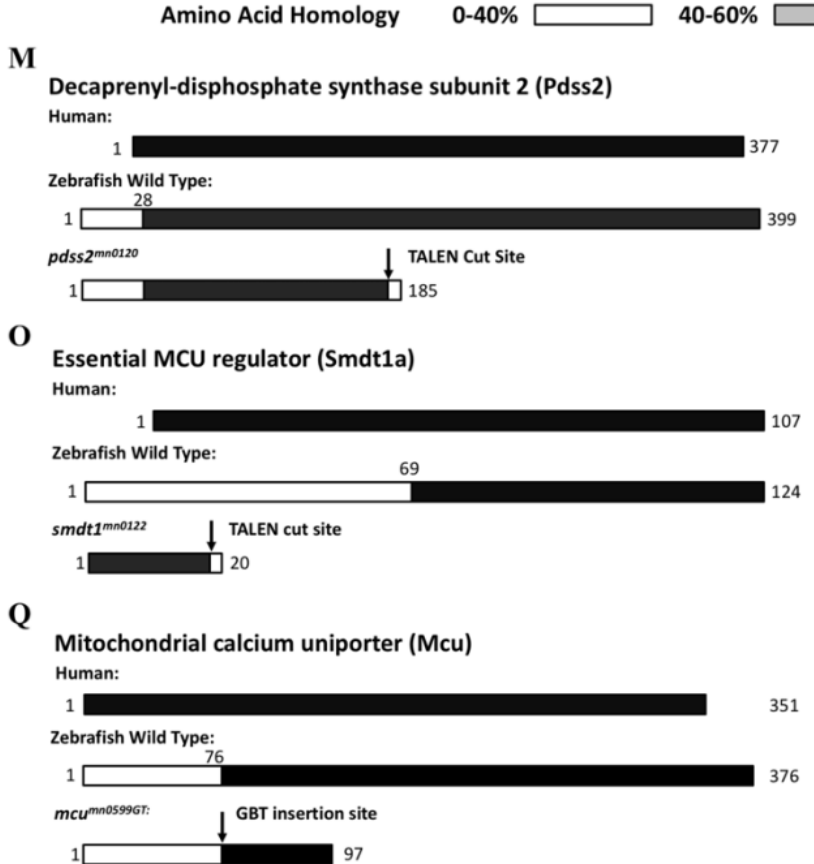

S

Leucine-rich pentatricopeptide repeat-containing (Lrpprc) Human:

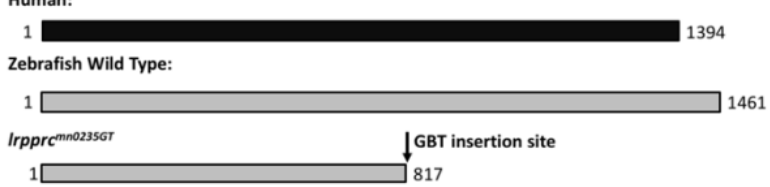

$\mathbf{U}$

28s ribosomal protein s18b (Mrps18b)

Human:

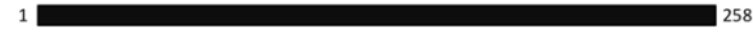

Zebrafish Wild Type:

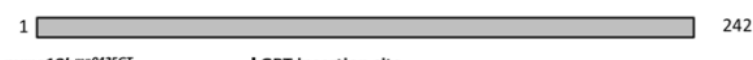

mrps18b

W

2-oxoglutarate dehydrogenase (Ogdhb)

Human:

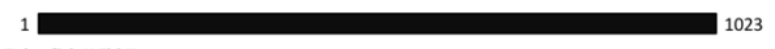

Zebrafish Wild Type:

$60-80 \% \square 80-100 \%$

$\mathbf{N}$

Mitochondrial calcium uptake protein 1 (Micu1)

Human:

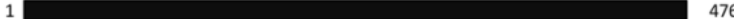

Zebrafish Wild Type:

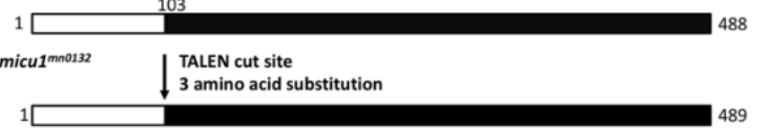

Mitochondrial calcium uniporter (Mcu)

Human:

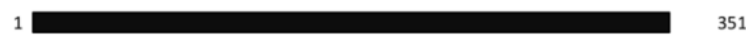

Zebrafish Wild Type:

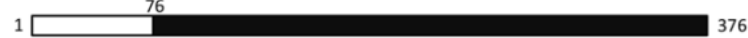

mcumno111 TALEN cut site *Exon 2 deleted

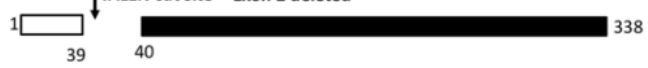

Thymidine kinase 2 (Tk2)

Human:

*Active site

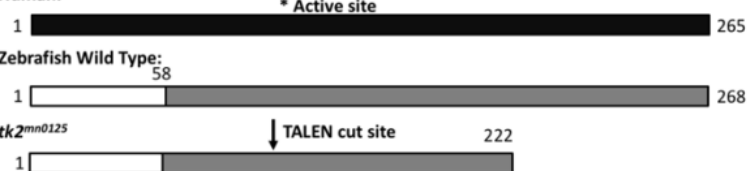

T Mitochondrial methionyl-tRNA formyltransferase (Mtfmt) Human:

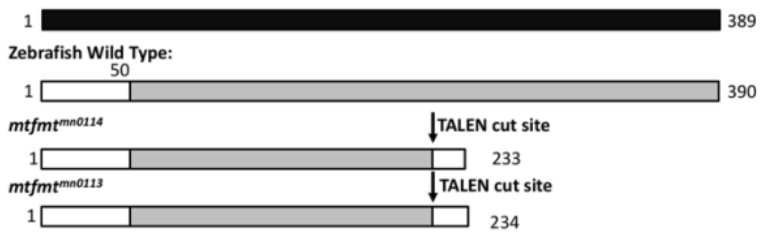

V Mitochondrial import inner membrane translocase subunit TIM50 (Timm50)

Human:

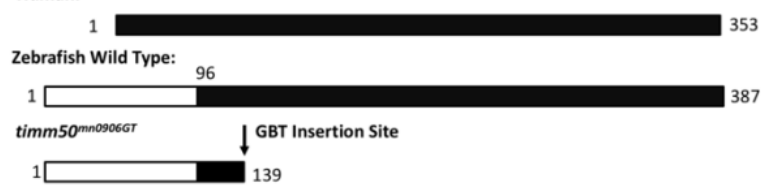

X Isocitrate Dehydrogenase (Idh2) Human:

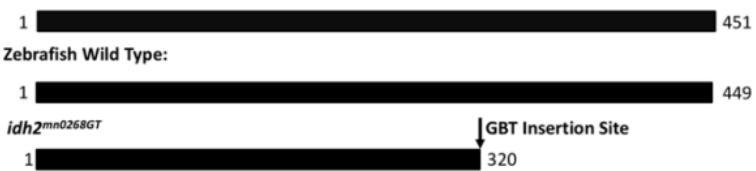

